\title{
Cross-Layer Design of Wireless Multihop Random Access Networks
}

\author{
Morteza Mardani, Student Member, IEEE, Seung-Jun Kim, Member, IEEE, and Georgios B. Giannakis, Fellow, IEEE
}

\begin{abstract}
Joint design of flow control, multipath routing, and random access control is considered for wireless multihop networks. Based on a network utility maximization formulation, Aloha persistence probabilities are optimized together with multicommodity end-to-end rates and per-link flow rates. Although the joint optimization of Aloha and flow control was previously tackled using a convex reformulation, adding the routing component renders the problem inherently nonconvex. To cope with this challenge, a successive convex approximation approach is taken to obtain a locally optimal solution efficiently. A parallelized distributed algorithm is developed, which scales well in the network size and exhibits low computational complexity. An online implementation is also proposed and tested. Numerical examples verify the novel design and highlight the performance advantage over state-of-the-art alternatives.
\end{abstract}

Index Terms-Multihop network, multipath routing, random access, successive convex approximation, utility maximization.

\section{INTRODUCTION}

$\mathbf{R}$ ANDOM access is a simple medium access control (MAC) solution that does not require centralized coordination, yet is effective for coping with wireless interference, especially when the traffic load is relatively light. Such features are desirable for wireless ad hoc networks including wireless sensor networks. Among the random access techniques, slotted Aloha is of much practical interest due to its implementation simplicity [4].

Recently, utility-optimal MAC design proved successful in mitigating some of the limitations associated with conventional random access protocols. The link persistence probabilities were optimized in [23] via a network utility maximization (NUM) formulation to improve fairness among interfering links compared to the existing IEEE 802.11 MAC. However, it was noted that the contention graph-based model for MAC used in [23] is overly simplified to fully capture the effects of probabilistic packet collisions and backoff mechanisms [15]. Thus, a precise probabilistic MAC model put forth in [29] was advocated to design utility-optimal MAC protocols through convex optimization in [15] and [22].

Manuscript received August 03, 2011; revised December 05, 2011; accepted January 24, 2012. Date of publication February 07, 2012; date of current version April 13, 2012. The associate editor coordinating the review of this manuscript and approving it for publication was Prof. Samson Lasaulce. This work was supported by NSF Grants CCF-0830480, ECCS-0824007, and ECCS-1002180. Part of the results in this paper was presented in the Signal Processing Applications in Wireless Communications Workshop, San Francisco, California, June 26-29, 2011

The authors are with the Department of Electrical and Computer Engineering, University of Minnesota, Minneapolis, MN 55455 USA (e-mail: morteza@umn.edu; seungjun@umn.edu; georgios@ece.umn.edu).

Color versions of one or more of the figures in this paper are available online at http://ieeexplore.ieee.org.

Digital Object Identifier 10.1109/TSP.2012.2187199
There is ample evidence corroborating the importance of cross-layer optimization in the wireless multihop setting [8], [11], [18], [30]. Engineering traffic flows through multihop routes is a crucial task in such networks. Optimal multipath routing establishes multiple explicit paths between source-destination pairs, and distributes appropriate amount of traffic on each of the paths. In wireless multihop networks employing random access, joint design of MAC and routing can improve the overall performance significantly, since "bottleneck areas" with frequent MAC collisions can be avoided by routing, while the nodes that contribute more on forwarding other nodes' traffic can be assigned with higher priorities in gaining access to the medium.

Joint optimization of flow control and Aloha-type MAC for multihop networks was investigated in [14], [16], [29], and [31]. The dual method is applied in [14], [29], and [31] to obtain distributed solutions, if end-to-end exchanges of control messages can be afforded. A backpressure-type algorithm is considered in [16], which dynamically sets the link access probability based on queue differentials. However, these works assume that routes are determined by separate mechanisms and do not consider path selection and traffic splitting among multiple paths.

Joint routing and MAC scheduling was tackled in [7], [13], and [24], where contention graph-based MAC models were used for simplicity. To circumvent the inherent nonconvexity, a harmonic mean of the multipath flow rates was considered in [28], resulting in approximately equal, yet suboptimal, flow splitting. It is noted that optimal multipath routing is a performance-critical traffic engineering issue, and efficient mechanisms such as MPLS are available to support such a feature in modern routing protocols [2], [10]. In essence, co-design of the wireless protocol stack including that of congestion control, routing, and random access has been undertaken in various practical settings [7], [24], [26], and is of particular interest in tactical settings [25].

The goal of this work is to obtain utility-optimal joint flow control, routing, and Aloha MAC parameters for wireless multihop networks in a distributed fashion. Multicommodity traffic and multipath routing are considered. An accurate probabilistic model for the random access MAC is adopted as in [15] and [29]. This model together with flow conservation constraints for routing leads to an inherently nonconvex problem, unlike the cases where multipath routing is not considered. To sidestep this hurdle, a successive convex approximation approach is employed to obtain an (at least locally) optimal solution efficiently. A convergent distributed algorithm is developed using the dual decomposition method. To much practical appeal, the optimal primal variables can be obtained at each node with very low complexity, and in some special cases of interest, in closed form. An online network control strategy is also developed, which obviates the need to wait for 


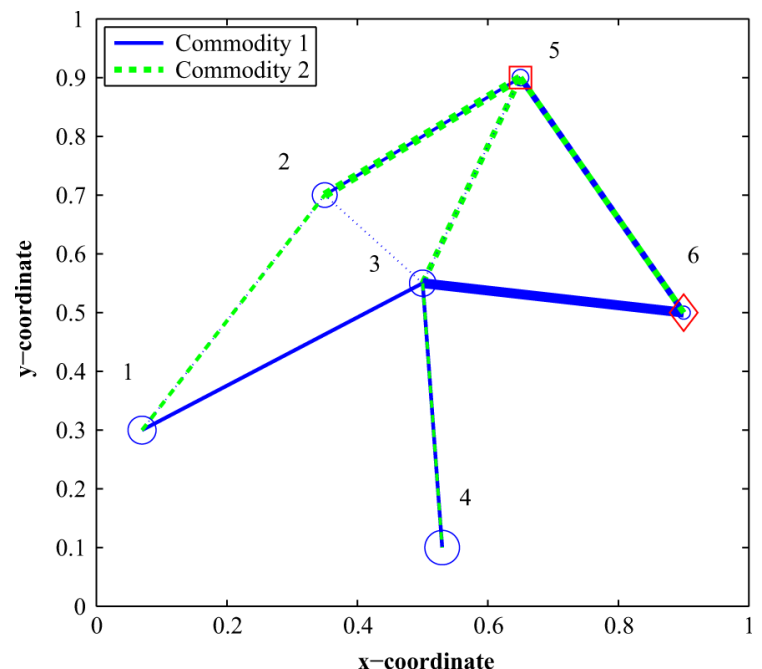

Fig. 1. Example network topology with the optimized persistence probabilities and flow rates.

an offline solution and allows tracking of (slowly) time-varying network parameters.

The rest of the paper is organized as follows. Section II deals with the system model and the NUM formulation. A successive convex approximation approach for the resulting nonconvex problem is discussed in Section III. Section IV develops a distributed algorithm as well as its online implementation. A contention graph-based approach for MAC design is developed in Section V. Numerical tests are provided in Section VI and conclusions in Section VII.

\section{System Model AND Problem Formulation}

A multihop wireless ad hoc network is considered with topology determined by a set of nodes $\mathcal{N}$, and a set of directed edges $\mathcal{L}$. Denote the transmitter node of link $\ell \in \mathcal{L}$ as $T x(\ell)$, and the receiver node as $R x(\ell)$. Let $\mathcal{L}_{\text {in }}(n) \triangleq\{\ell \in \mathcal{L} \mid R x(\ell)=n\}$ and $\mathcal{L}_{\text {out }}(n) \triangleq\{\ell \in \mathcal{L} \mid T x(\ell)=n\}$ represent the sets of edges corresponding to incoming and outgoing links of node $n$, respectively. The network is assumed to carry a set $\mathcal{I}$ of traffic "commodities," where commodity $i$ generated by a source node in set $\mathcal{S}_{i}$ is sent to the destination nodes in set $\mathcal{D}_{i}$. In general, node $n$ generates commodity $i$ traffic, absorbs it, or purely relays it to its neighbors. Let $\mathcal{I}_{n} \triangleq\left\{i \mid n \in \mathcal{S}_{i}\right\}$ denote the set of commodities generated by node $n$, and $\mathcal{J}_{n} \triangleq\left\{i \mid n \notin \mathcal{D}_{i}\right\}$ the set of commodities not absorbed by node $n$. Obviously, $\mathcal{J}_{n}=\mathcal{I}$ for the nodes that are not the sinks of any commodity.

Due to the broadcast nature of the wireless interface, a link whose receiver is in the proximity of an unintended co-channel transmitter is interfered if the corresponding reception and transmission occur simultaneously. Let $\mathcal{N}_{\text {to }}(\ell)$ be the set of nodes whose transmission interferes with (the reception of) link $\ell$. Similarly, let $\mathcal{L}_{\text {from }}(n)$ denote the set of links that are interfered by the transmission of node $n$. Note that if $\ell \in \mathcal{L}_{\text {from }}(n)$, then $n \in \mathcal{N}_{\text {to }}(\ell)$, and vice versa. For example, consider a network of six nodes, shown in Fig. 1, where all links are bidirectional. The communication and the interference distances are assumed to be identical. Then, $\mathcal{L}_{\text {from }}(4)=\{(1,3),(2,3),(4,3),(5,3),(6,3)\}$ and $\mathcal{N}_{\text {to }}((3,1))=\{1,2\}$.

Hardware limitations (e.g., the coupling between transmit-and receive-chains) dictate that each node can either transmit to, or receive from at most a single adjacent node. Moreover, it is assumed that the channel between any pair of nodes is quasi-static - a reasonable assumption in a low mobility scenario - although this assumption can be partially relieved when considering on-line implementation; cf. Section IV-D. Similar to [7] and [15], it is also assumed that fading impairments are fully mitigated by the physical layer. Thus, the interference relations are time-invariant, and packet drops are entirely due to collisions at the MAC layer.

To mitigate packet collisions due to interference, slotted Aloha is adopted for random access [4]. Let $\pi_{n}$ denote the persistence probability of node $n$; that is, node $n$ transmits with probability $\pi_{n}$, provided that there is a packet in its queue. Given that node $n$ transmits, the probability of forwarding a packet through link $\ell \in \mathcal{L}_{\text {out }}(n)$ is denoted by $q_{\ell}$, where $\sum_{\ell \in \mathcal{L}_{\text {out }}(n)} q_{\ell}=1$. Thus, the access probability of link $\ell$ is given by $p_{\ell}=q_{\ell} \pi_{n}$ with $\sum_{\ell \in \mathcal{L}_{\text {out }}(n)} p_{\ell}=\pi_{n}$. Define vectors $\boldsymbol{\pi} \triangleq\left[\pi_{n}\right]_{n \in \mathcal{N}}$ and $\mathbf{p} \triangleq\left[p_{\ell}\right]_{\ell \in \mathcal{L}}$.

Let $c_{\ell}$ represent the raw physical layer rate of link $\ell$ during data transmission assuming no collision. Suppose also that the nodes are fully backlogged, and multi-packet reception is not allowed [12]. Since nodes transmit independently, the average rate $C_{\ell}$ delivered by the MAC to higher layers can be expressed as

$$
C_{\ell}(\mathbf{p}, \boldsymbol{\pi})=c_{\ell} p_{\ell} \prod_{n \in \mathcal{N}_{\text {to }}(\ell)}\left(1-\pi_{n}\right), \quad \ell \in \mathcal{L} .
$$

A source node $n \in \mathcal{S}_{i}$ generates commodity $i$ traffic at an average rate of $s_{n}^{(i)} \geq 0$ for $i \in \mathcal{I}_{n}$. Multipath routing based on traffic splitting is considered so that the generated traffic can exploit multiple routes to reach the destinations in $\mathcal{D}_{i}$ [2], [10]. Node $m$ in one of the paths forwards traffic to the neighbors through its outgoing links $\ell \in \mathcal{L}_{\text {out }}(m)$ at average flow rate $r_{\ell}^{(i)} \geq 0$.

\section{Define vectors}

$$
\mathbf{s} \triangleq\left[s_{n}^{(i)}\right]_{n \in \mathcal{S}_{i}, i \in \mathcal{I}} \text { and } \mathbf{r} \triangleq\left[r_{\ell}^{(i)}\right]_{\ell \in \mathcal{L}_{\text {out }}(n), n \in \mathcal{N} \backslash \mathcal{D}_{i}, i \in \mathcal{I}}
$$

formed by the input rates of all sources and the flow rates of all links in the network, except those emanating from the sinks of corresponding commodities. Note that the links emanating from the sinks of a commodity cannot carry the traffic of that commodity as the sinks will absorb all the traffic by definition. In the same token, let $\mathcal{L}_{\text {in }}^{(i)}(n) \triangleq\left\{\ell \in \mathcal{L}_{\text {in }}(n) \mid T x(\ell) \notin \mathcal{D}_{i}\right\}$ denote the set of incoming links of node $n$, excluding those that emanate from a destination node for commodity $i$ traffic. Similarly, define $\mathcal{L}_{\text {out }}^{(i)}(n) \triangleq\left\{\ell \in \mathcal{L}_{\text {out }}(n) \mid R x(\ell) \notin \mathcal{D}_{i}\right\}$ as the set of outgoing links of node $n$, except those entering a destination of the $i$ th traffic commodity. For example, consider again the network in Fig. 1, which carries two traffic commodities (i.e., $\mathcal{I}=\{1,2\}$ ). Nodes marked by diamonds and squares represent the destination nodes of commodities 1 and 2, respectively. Nodes 1 through 4 generate both commodities, while nodes 5 and 6 generate commodities 1 and 2, respectively. Then, $\mathcal{L}_{\text {out }}^{(1)}(5)=\{(5,2),(5,3)\}$ and $\mathcal{L}_{\text {in }}^{(1)}(5)=\{(2,5),(3,5)\}$.

For queue stability, the flow conservation law of the network, expressed as

$$
\sum_{\ell \in \mathcal{L}_{\text {out }}(n)} r_{\ell}^{(i)} \geq \sum_{\ell \in \mathcal{L}_{\mathrm{in}}^{(i)}(n)} r_{\ell}^{(i)}+s_{n}^{(i)} \mathbb{1}_{\left\{n \in \mathcal{S}_{i}\right\}}, n \in \mathcal{N} \backslash \mathcal{D}_{i}, i \in \mathcal{I}
$$


must hold, where $\mathbb{1}_{A}$ denotes the indicator function, taking value 1 if $A$ is true, and 0 otherwise. The r.h.s. of (2) corresponds to the commodity $i$ traffic entering node $n$, where the source variable $s_{n}^{(i)}$ appears only when node $n$ is a source node. Note that by using $\mathcal{L}_{\text {in }}^{(i)}(n)$ instead of $\mathcal{L}_{\text {in }}(n)$ in (2), the flows emanating from the destination nodes of commodities are excluded (since they must be zero by the definition of destination nodes).

A NUM formulation will be sought to find the optimal random access MAC parameters $\mathbf{p}$ and $\boldsymbol{\pi}$, the multihop routing variables $\mathbf{r}$, and the source rate variables $\mathbf{s}$. Source node $n$ that can deliver commodity $i$ traffic to any destination in $\mathcal{D}_{i}$ at rate $s_{n}^{(i)}$, is awarded utility $U_{n}^{(i)}\left(s_{n}^{(i)}\right)$. The class of concave and monotonically increasing utility functions adopted in this work is

$$
U_{n}^{(i)}(x)=\left\{\begin{array}{ll}
\left(1-\beta_{n}^{(i)}\right)^{-1} x^{\left(1-\beta_{n}^{(i)}\right)}, & \beta_{n}^{(i)} \neq 1 \\
\log x, & \beta_{n}^{(i)}=1
\end{array},\right.
$$

for $n \in \mathcal{S}_{i}, i \in \mathcal{I}$ where $\beta_{n}^{(i)} \geq 0$ is a fairness-controlling parameter. For example, setting $\beta_{n}^{\overline{(i)}}=1 \forall n, i$ amounts to proportional fairness; $\beta_{n}^{(i)}=2 \forall n, i$, harmonic-mean fairness; and, $\beta_{n}^{(i)}=\infty \forall n, i$, max-min fairness, among all source nodes and commodities [21]. The optimization problem that maximizes the weighted total network utility is formulated as

$$
\max _{\substack{\mathbf{p} \succeq \mathbf{0}, \mathbf{0} \preceq \mathbf{\pi} \preceq \mathbf{1}, \mathbf{s} \succeq \mathbf{0} \\ \mathbf{r}_{\min } \preceq \mathbf{r} \preceq \mathbf{r}_{\max }}} \sum_{i \in \mathcal{I}} \sum_{n \in \mathcal{S}_{i}} w_{n}^{(i)} U_{n}^{(i)}\left(s_{n}^{(i)}\right)
$$

$$
\begin{aligned}
& \text { s. to } \sum_{i \in \mathcal{J}_{n}} r_{\ell}^{(i)} \leq c_{\ell} p_{\ell} \prod_{m \in \mathcal{N}_{\text {to }}(\ell)}\left(1-\pi_{m}\right), \\
& \sum_{\ell \in \mathcal{L}_{\text {out }}(n)} p_{\ell}=\pi_{n}, n \in \mathcal{N} \begin{array}{l}
\ell \text { out } \\
\quad(n), n \in \mathcal{N}
\end{array} \\
& \sum_{\ell \in \mathcal{L}_{\text {out }}(n)} r_{\ell}^{(i)} \geq \sum_{\ell \in \mathcal{L}_{\text {in }}^{(i)}(n)} r_{\ell}^{(i)}+s_{n}^{(i)} \mathbb{1}_{\left\{n \in \mathcal{S}_{i}\right\}}, \\
& n \in \mathcal{N} \backslash \mathcal{D}_{i}, i \in \mathcal{I}
\end{aligned}
$$

where the constants $\left\{w_{n}^{(i)}\right\}$ are positive weights adjusting the importance of different nodes and commodities; $\mathbf{0}$ and $\mathbf{1}$ are vectors with all elements equal to zero and one, respectively; and vectors $\mathbf{r}_{\text {min }} \triangleq\left[r_{\ell, \min }^{(i)}\right]_{\ell \in \mathcal{L}_{\text {out }}(n), n \in \mathcal{N} \backslash \mathcal{D}_{i}, i \in \mathcal{I}}$ and $\mathbf{r}_{\text {max }} \triangleq\left[r_{\ell \text { max }}^{(i)}\right]_{\ell \in \mathcal{L}_{\text {out }}(n), n \in \mathcal{N} \backslash \mathcal{D}_{i}, i \in \mathcal{I}}$ denote the minimum and maximum average rates of the links, respectively. To bypass a technicality that will become clear later, it is assumed that $\mathbf{r}_{\text {min }} \succ \mathbf{0}$. However, as the entries of $\mathbf{r}_{\text {min }}$ can take arbitrarily small values, such an assumption is practically inconsequential. Constraint (5) states that the flow rate over each link $\ell \in \mathcal{L}$ cannot exceed the achievable rate delivered by the MAC layer. Constraint (6) relates the link access probabilities to the node access probabilities as previously discussed. Constraint (7) ensures queue stability. To be precise, (7) is a necessary condition for queue stability. A sufficient condition can be readily obtained by adding a small positive constant $\sigma$ on the r.h.s. of (7). For the sake of simplicity, this subtlety is neglected in this work.
Problem (P1) is not convex due to (5), and thus hard to solve even in a centralized fashion. In the next section, an approach to obtain an (at least locally) optimal solution is described.

\section{SuCCESSIVE CONVEX APPROXIMATION}

When multipath routes are not optimized jointly, convex reformulation of (P1) is possible through a change of variables $\tilde{r}_{\ell}^{(i)}:=\log r_{\ell}^{(i)}$ and $\tilde{s}_{n}^{(i)}:=\log s_{n}^{(i)}$ after taking logarithms on both sides of (5). However, in the present case of jointly optimal MAC, multipath routing, and flow control, this change of variables also transforms the flow conservation constraints (7) into nonconvex constraints, and the problem remains nonconvex.

To find a locally optimal solution to (P1), we adopt the said change of variables, but mitigate the resulting nonconvexity in (7) via successive convex approximation [20]. To this end, consider first rearranging (7) to obtain a set of constraints on the ratios of posynomials [1], as

$$
\frac{s_{n}^{(i)} \mathbb{1}_{\left\{n \in \mathcal{S}_{i}\right\}}+\sum_{\ell \in \mathcal{L}_{\text {in }}^{(i)}(n)} r_{\ell}^{(i)}}{\sum_{\ell \in \mathcal{L}_{\text {out }}(n)} r_{\ell}^{(i)}} \leq 1, n \in \mathcal{N} \backslash \mathcal{D}_{i}, i \in \mathcal{I}
$$

where the denominator is positive since $\mathbf{r}_{\min } \succ \mathbf{0}$. The 1.h.s. of (8) is still a nonconvex function, which motivates the ensuing iterative approach based on successive convex approximation. Specifically, the single condensation method is employed to approximate the ratio of posynomials by a posynomial, as explained next; see also [1] and [9].

Given the iterates $\left\{r_{\ell}^{(i)}(k-1)\right\}$ from (previous) iteration $k-$ 1 , the denominator in the 1.h.s. of (8) can be lower bounded by a monomial as

$$
\sum_{\ell \in \mathcal{L}_{\text {out }}(n)} r_{\ell}^{(i)} \geq \prod_{\ell \in \mathcal{L}_{\text {out }}(n)}\left(\frac{r_{\ell}^{(i)}}{\alpha_{\ell}^{(i)}(k)}\right)^{\alpha_{\ell}^{(i)}(k)}
$$

where $\left\{\alpha_{\ell}^{(i)}(k)\right\}$ with $\sum_{\ell \in \mathcal{L}_{\text {out }}(n)} \alpha_{\ell}^{(i)}(k)=1$ are constants obtained recursively from $\left\{r_{\ell}^{(i)}(k-1)\right\}$ as

$$
\begin{aligned}
\alpha_{\ell}^{(i)}(k) & =\frac{r_{\ell}^{(i)}(k-1)}{\sum_{\ell \in \mathcal{L}_{\text {out }}(n)} r_{\ell}^{(i)}(k-1)}, \\
\ell & \in \mathcal{L}_{\text {out }}(n), n \in \mathcal{N} \backslash \mathcal{D}_{i}, i \in \mathcal{I} .
\end{aligned}
$$

It has been shown (see, e.g., [9]) that the posynomial obtained by replacing the denominator of the 1.h.s. of (8) by the lower-bound in (9) satisfies the conditions for convergence of the successive convex approximation method [20]. For example, it can be easily verified that equality is achieved in (9) at $r_{\ell}^{(i)}=r_{\ell}^{(i)}(k-1) \forall \ell, i$. Then, using the change of variables $\tilde{r}_{\ell}^{(i)}:=\log r_{\ell}^{(i)}$, and $\tilde{s}_{n}^{(i)}:=\log s_{n}^{(i)}$, the constraints in (8) are replaced by tighter convex surrogates given by

$$
\begin{aligned}
\log \left(e^{\tilde{s}_{n}^{(i)}} \mathbb{1}_{\left\{n \in \mathcal{S}_{i}\right\}}+\sum_{\ell \in \mathcal{L}_{\text {in }}^{(i)}(n)} e^{\tilde{r}_{\ell}^{(i)}}\right)-\sum_{\ell \in \mathcal{L}_{\text {out }}(n)} \alpha_{\ell}^{(i)}(k) \\
\quad \times\left(\tilde{r}_{\ell}^{(i)}-\log \alpha_{\ell}^{(i)}(k)\right) \leq 0, n \in \mathcal{N} \backslash \mathcal{D}_{i}, i \in \mathcal{I} .
\end{aligned}
$$


TABLE I

ALGORITHM TO OBTAIN A KKT SOLUTION TO (P1)

\begin{tabular}{|l}
\hline Step 0) Set $k=1$. Initialize $(\mathbf{p}(0), \boldsymbol{\pi}(0), \tilde{\mathbf{r}}(0), \tilde{\mathbf{s}}(0))$ \\
to a feasible point of $(\mathrm{P} 1)$. \\
Step 1) Compute $\left\{\alpha_{\ell}^{(i)}(k)\right\}$ via $(10)$ and solve $(\mathrm{P} 2)$ \\
for $(\mathbf{p}(k), \boldsymbol{\pi}(k), \tilde{\mathbf{r}}(k), \tilde{\mathbf{s}}(k))$ either in a centralized fashion, \\
or in a distributed fashion through the algorithm in Table II. \\
Step 2) Stop if converged \\
and output $\left(\mathbf{p}(k), \boldsymbol{\pi}(k), \mathbf{r}(k)=e^{\tilde{\mathbf{r}}(k)}, \mathbf{s}(k)=e^{\tilde{\mathbf{s}}(k)}\right)$ \\
otherwise set $k \leftarrow k+1$, and go to Step 1$)$
\end{tabular}

Upon defining $\tilde{\mathbf{r}}, \tilde{\mathbf{s}}, \tilde{\mathbf{r}}_{\min }$ and $\tilde{\mathbf{r}}_{\max }$ as the vectors obtained by taking entrywise logarithm of $\mathbf{r}, \mathbf{s}, \mathbf{r}_{\text {min }}$ and $\mathbf{r}_{\text {max }}$, respectively, and letting $\tilde{U}_{n}^{(i)}\left(\tilde{s}_{n}^{(i)}\right) \triangleq U_{n}^{(i)}\left(e^{\tilde{s}_{n}^{(i)}}\right)$ and $\tilde{c}_{\ell} \triangleq \log c_{\ell}$, the problem to solve at the $k$ th iteration of the successive approximation algorithm is

$$
\max _{\substack{\tilde{\mathbf{s}}, \mathbf{p} \succeq \mathbf{0}, \mathbf{0} \preceq \boldsymbol{\pi} \preceq \mathbf{1} \\ \tilde{\mathbf{r}} \min }} \sum_{i \in \mathcal{r} \preceq \tilde{\mathbf{r}}_{\max }} \sum_{n \in \mathcal{S}_{i}} w_{n}^{(i)} \tilde{U}_{n}^{(i)}\left(\tilde{s}_{n}^{(i)}\right)
$$

s. to: (6), (11), and

$$
\begin{aligned}
& \log \left(\sum_{i \in \mathcal{J}_{n}} e^{\tilde{r}_{\ell}^{(i)}}\right)-\tilde{c}_{\ell}-\log p_{\ell}-\sum_{m \in \mathcal{N}_{\mathrm{to}}(\ell)} \log \left(1-\pi_{m}\right) \\
& \leq 0, \ell \in \mathcal{L}_{\text {out }}(n), n \in \mathcal{N}
\end{aligned}
$$

where (13) is obtained by taking logarithm of both sides of (5) after the change of variables.

Proposition 1: If $\beta_{n}^{(i)}$ in (3) is chosen such that $\beta_{n}^{(i)} \geq$ $1, \forall n, i$, then (P2) is convex.

Proof: With $\beta_{n}^{(i)} \geq 1$, it is straightforward to verify that the second derivative of $\tilde{U}_{n}^{(i)}\left(\tilde{s}_{n}^{(i)}\right)$ with respect to $\tilde{s}_{n}^{(i)}$ is nonpositive [15]. Thus, the objective in (12) is concave. The l.h.s. of (11) and (13) are convex because the log-sum-exp function is convex [5]. Since the remaining constraints are linear, the proof is complete.

With the choice $\beta_{n}^{(i)} \geq 1 \forall n, i$, Table I presents an iterative procedure that yields a KKT solution to (P1). Convergence of the successive approximation algorithm based on the single condensation method, such as the one in Table I, has been established in [9]; see also [20]. In Step 0), the feasible starting point can be obtained, e.g., by choosing $0<\pi_{n} \leq 1$ and setting $p_{\ell}=\frac{\pi_{n}}{\left|\mathcal{L}_{\text {out }}(n)\right|}$ for $\ell \in \mathcal{L}_{\text {out }}(n), n \in \mathcal{N}$, in (P1). Provided that the entries of $\mathbf{r}_{\text {min }}$ are small enough, feasible values for $\left\{r_{\ell}^{(i)}\right\}$ and $\left\{s_{n}^{(i)}\right\}$ can be easily found. Step 1) can be performed using a centralized solver, as (P2) is convex. However, a distributed algorithm is often desired due to its scalability and robustness attributes. This is the subject of the next section.

\section{Distributed Solution}

Problem (P2) is not amenable to distributed implementation in its present form owing to the coupling among the flow variables for different nodes in (11). To effect decoupling, a set of auxiliary variables is introduced to maintain local copies of the outgoing $(\log )$ flow rates at each node. Additional constraints are then imposed to enforce that the copies indeed match the originals. Then, the dual method can be employed to decompose the overall problem into smaller subproblems that can be solved individually at each node, coordinated by Lagrange multipliers.

Since the flow conservation constraints, and subsequently the constraints (11), are not imposed for the destination nodes of each traffic commodity $i \in \mathcal{I}$, care must be taken. That is, the copies need not be made for the flows of commodities that enter the destination nodes of the commodities. For notational convenience, let $\mathcal{A}_{n} \triangleq\left\{(\ell, i) \mid \ell \in \mathcal{L}_{\text {in }}^{(i)}(n), i \in \mathcal{J}_{n}\right\} \equiv\{(\ell, i) \mid \ell \in$ $\left.\mathcal{L}_{\text {in }}(n), T x(\ell) \notin \mathcal{D}_{i}, R x(\ell) \notin \mathcal{D}_{i}, i \in \mathcal{I}\right\}$ denote the set of link-commodity pairs, where the link enters node $n$, but is not connected to a destination node of the commodity. Similarly, define $\mathcal{B}_{n} \triangleq\left\{(\ell, i) \mid \ell \in \mathcal{L}_{\text {out }}(n), T x(\ell) \notin \mathcal{D}_{i}, R x(\ell) \in \mathcal{D}_{i}, i \in\right.$ $\mathcal{I}\}$ and $\mathcal{C}_{n} \triangleq\left\{(\ell, i) \mid \ell \in \mathcal{L}_{\text {out }}(n), T x(\ell) \notin \mathcal{D}_{i}, R x(\ell) \notin\right.$ $\left.\mathcal{D}_{i}, i \in \mathcal{I}\right\}$ for the outgoing links of node $n$. For example, it can be seen in Fig. 1 that $\mathcal{A}_{6}=\{((3,6), 2)\}, \mathcal{B}_{6}=\{((6,5), 2)\}$, and $\mathcal{C}_{6}=\{((6,3), 2)\}$.

Then, introduce a set of auxiliary variables $\hat{\mathbf{v}} \triangleq$ $\left[\tilde{v}_{\ell}^{(i)}\right]_{(\ell, i) \in \mathcal{C}_{n}, n \in \mathcal{N}}$ that serves as a copy of the set of outgoing log flow rate variables $\hat{\mathbf{r}} \triangleq\left[\tilde{r}_{\ell}^{(i)}\right]_{(\ell, i) \in \mathcal{C}_{n}, n \in \mathcal{N}}$, where the flows entering the destination nodes of the corresponding commodities are excluded from $\tilde{\mathbf{r}}$. Define also $\hat{\mathbf{r}}_{\text {min }} \triangleq\left[\tilde{r}_{\ell, \min }^{(i)}\right]_{(\ell, i) \in \mathcal{C}_{n}, n \in \mathcal{N}}$ and $\hat{\mathbf{r}}_{\text {max }} \triangleq\left[\tilde{r}_{\ell \text { max }}^{(i)}\right]_{(\ell, i) \in \mathcal{C}_{n}, n \in \mathcal{N}}$.

An important practical issue in using the dual method is the ability to obtain the primal optimal variables efficiently, most preferably in closed form [3, p. 593]. In fact, it can be shown that a single-commodity version of (P2) admits closed-form solutions for the primal variables when $\beta_{n}^{(1)}=1$; and very efficient solutions involving a bisection search when $\beta_{n}^{(1)}>1$ [19]. However, such merits do not translate to the present multi-commodity case, because variables corresponding to distinct traffic commodities become coupled in the log-sum-exp term in (13). Fortunately, replacing the MAC constraints (5) by the following equivalent set of constraints, through the additional auxiliary variables $\left\{a_{\ell}^{(i)}\right\}$, yields efficient primal solutions (see also [31]):

$$
\begin{aligned}
& r_{\ell}^{(i)} \leq a_{\ell}^{(i)} c_{\ell} p_{\ell} \prod_{m \in \mathcal{N}_{\text {to }}(\ell)}\left(1-\pi_{m}\right), \\
& \quad \ell \in \mathcal{L}_{\text {out }}(n), n \in \mathcal{N} \backslash \mathcal{D}_{i}, i \in \mathcal{I} \\
& \quad 0 \leq a_{\ell}^{(i)} \leq 1, \quad \ell \in \mathcal{L}_{\text {out }}(n), n \in \mathcal{N} \backslash \mathcal{D}_{i}, i \in \mathcal{I} \\
& \quad \sum_{i \in \mathcal{J}_{n}} a_{\ell}^{(i)}=1, \quad \ell \in \mathcal{L}_{\text {out }}(n), n \in \mathcal{N} .
\end{aligned}
$$

Here, $a_{\ell}^{(i)}$ can be interpreted as the fraction of the MAC achievable rate on link $\ell$ allocated to commodity $i$ traffic. Another challenge with the dual method is that the primal optimal variables recovered from the dual optimal ones may be infeasible if the primal variables are not uniquely determined [3, Ch.6]. To enforce uniqueness, one can add regularization terms to the objective in (12). Letting $\check{\mathbf{r}} \triangleq\left[\tilde{r}_{\ell}^{(i)}\right]_{(\ell, i) \in \mathcal{B}_{n}, n \in \mathcal{N}}$ and defining $\check{\mathbf{r}}_{\text {min }}$ and $\check{\mathbf{r}}_{\text {max }}$ likewise, the useful regularization terms with the magnitude controlled by a small constant $\epsilon>0$, are (see also [30])

$$
R_{\epsilon}(\hat{\mathbf{v}}, \check{\mathbf{r}}) \triangleq-\epsilon \sum_{n \in \mathcal{N}}\left\{\sum_{(\ell, i) \in \mathcal{C}_{n}}\left(\tilde{v}_{\ell}^{(i)}\right)^{2}+\sum_{(\ell, i) \in \mathcal{B}_{n}}\left(\tilde{r}_{\ell}^{(i)}\right)^{2}\right\} .
$$


Upon defining $\tilde{z}_{\ell}^{(i)} \triangleq \tilde{v}_{\ell}^{(i)} \mathbb{1}_{\left\{R x(\ell) \notin \mathcal{D}_{i}\right\}}+\tilde{r}_{\ell}^{(i)} \mathbb{1}_{\left\{R x(\ell) \in \mathcal{D}_{i}\right\}}$, and incorporating the preceding discussions, it is possible to reformulate (P2) as

(P3)

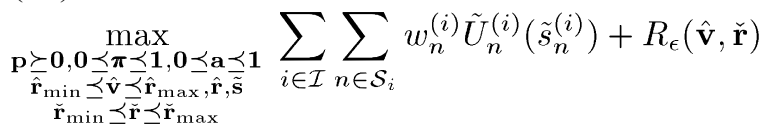

s. to: (6), (16), and

$$
\begin{aligned}
& \tilde{r}_{\ell}^{(i)}-\log a_{\ell}^{(i)}-\tilde{c}_{\ell}-\log p_{\ell}-\sum_{m \in \mathcal{N}_{\text {to }}(\ell)} \log \left(1-\pi_{m}\right) \leq 0, \\
& \ell \in \mathcal{L}_{\text {out }}(n), i \in \mathcal{J}_{n}, n \in \mathcal{N} \\
& \log \left(e^{\tilde{s}_{n}^{(i)}} \mathbb{1}_{\left\{n \in \mathcal{S}_{i}\right\}}+\sum_{\ell \in \mathcal{L}_{\text {in }}^{(i)}(n)} e^{\tilde{r}_{\ell}^{(i)}}\right)-\sum_{\ell \in \mathcal{L}_{\text {out }}(n)} \alpha_{\ell}^{(i)}(k) \\
& \times\left(\tilde{z}_{\ell}^{(i)}-\log \alpha_{\ell}^{(i)}(k)\right) \leq 0, i \in \mathcal{J}_{n}, n \in \mathcal{N} \\
& \tilde{v}_{\ell}^{(i)}=\tilde{r}_{\ell}^{(i)}, \quad(\ell, i) \in \mathcal{C}_{n}, n \in \mathcal{N}
\end{aligned}
$$

where the fact that $\left\{(n, i) \mid n \in \mathcal{N} \backslash \mathcal{D}_{i}, i \in \mathcal{I}\right\}$ is equivalent to $\left\{(n, i) \mid i \in \mathcal{J}_{n}, n \in \mathcal{N}\right\}$, was used in (18) and (19). Arguing as in Proposition 1, one can show that (P3) is convex when $\beta_{n}^{(i)} \geq 1 \forall n, i$. Thus, assuming that strong duality holds for (P3), the dual method can be employed to solve (P3) optimally in a distributed fashion [3, Ch. 6].

Introduce Lagrange multipliers

$$
\lambda \triangleq\left[\lambda_{\ell}^{(i)}\right]_{\ell \in \mathcal{L}_{\text {out }}(n), i \in \mathcal{J}_{n}, n \in \mathcal{N}}
$$

with $\boldsymbol{\lambda} \succeq \mathbf{0}$ and $\boldsymbol{\gamma} \triangleq\left[\gamma_{\ell}^{(i)}\right]_{(\ell, i) \in \mathcal{C}_{n}, n \in \mathcal{N}}$ to relax constraints (18) and (20), respectively. To solve (P3) in a distributed manner, define variables local to each node $n \in \mathcal{N}$. First, define node $n$ 's primal variables $\mathbf{p}_{n} \triangleq\left[p_{\ell}\right]_{\ell \in \mathcal{L}_{\text {out }}(n)}, \mathbf{a}_{n} \triangleq$ $\left[a_{\ell}^{(i)}\right]_{\ell \in \mathcal{L}_{\text {out }}(n), i \in \mathcal{J}_{n}}, \tilde{\mathbf{s}}_{n} \triangleq\left[\tilde{s}_{n}^{(i)}\right]_{i \in \mathcal{I}_{n}}, \tilde{\mathbf{r}}_{n} \triangleq\left[\tilde{r}_{\ell}^{(i)}\right]_{(\ell, i) \in \mathcal{A}_{n} \cup \mathcal{B}_{n}}$, and $\hat{\mathbf{v}}_{n} \triangleq\left[\tilde{v}_{\ell}^{(i)}\right]_{(\ell, i) \in \mathcal{C}_{n}}$. Collect also the Lagrange multipliers related to node $n$ to form $\lambda_{n} \triangleq\left[\lambda_{\ell}^{(i)}\right]_{\ell \in \mathcal{L}_{\text {out }}(n), i \in \mathcal{J}_{n}}$, $\boldsymbol{\gamma}_{n} \triangleq\left[\gamma_{\ell}^{(i)}\right]_{(\ell, i) \in \mathcal{A}_{n} \cup \mathcal{C}_{n}}, \tilde{\boldsymbol{\lambda}}_{n} \triangleq\left[\lambda_{\ell}^{(i)}\right]_{(\ell, i) \in \mathcal{A}_{n} \cup \mathcal{B}_{n}}$, and $\bar{\lambda}_{n} \triangleq\left[\lambda_{\ell}\right]_{\ell \in \mathcal{L}_{\text {from }}(n)}$, where $\lambda_{\ell} \triangleq \sum_{i \in \mathcal{J}_{n}} \lambda_{\ell}^{(i)}$. Then, the partial Lagrangian can be written as

$$
\begin{aligned}
L(\mathbf{p}, \boldsymbol{\pi}, \mathbf{a}, \tilde{\mathbf{r}}, \hat{\mathbf{v}}, \tilde{\mathbf{s}}, \boldsymbol{\lambda}, \boldsymbol{\gamma})=\sum_{n \in \mathcal{N}}\left[L_{n}^{\mathrm{MAC}}\left(\mathbf{p}_{n}, \pi_{n}, \mathbf{a}_{n}, \boldsymbol{\lambda}_{n}, \overline{\boldsymbol{\lambda}}_{n}\right)\right. \\
\left.+L_{n}^{\mathrm{high}}\left(\tilde{\mathbf{s}}_{n}, \tilde{\mathbf{r}}_{n}, \hat{\mathbf{v}}_{n}, \tilde{\boldsymbol{\lambda}}_{n}, \boldsymbol{\gamma}_{n}\right)\right]
\end{aligned}
$$

where

$$
\begin{aligned}
L_{n}^{\mathrm{MAC}}(\cdot) \triangleq & \sum_{\ell \in \mathcal{L}_{\text {out }}(n)}\left[\lambda_{\ell} \tilde{c}_{\ell}+\lambda_{\ell} \log p_{\ell}\right]+\log \left(1-\pi_{n}\right) \\
& \times \sum_{\ell \in \mathcal{L}_{\text {from }}(n)} \lambda_{\ell}+\sum_{\ell \in \mathcal{L}_{\text {out }}(n)} \sum_{i \in \mathcal{J}_{n}} \lambda_{\ell}^{(i)} \log a_{\ell}^{(i)}
\end{aligned}
$$

and

$$
\begin{aligned}
& L_{n}^{\mathrm{high}}(\cdot) \\
& \triangleq \sum_{i \in \mathcal{I}_{n}} w_{n}^{(i)} \tilde{U}_{n}^{(i)}\left(\tilde{s}_{n}^{(i)}\right)-\sum_{i \in \mathcal{J}_{n}}\left\{\sum_{\ell \in \mathcal{L}_{\text {out }}^{(i)}(n)}\left[\gamma_{\ell}^{(i)} \tilde{v}_{\ell}^{(i)}+\epsilon\left(\tilde{v}_{\ell}^{(i)}\right)^{2}\right]\right. \\
& \left.+\sum_{\ell \in \mathcal{L}_{\text {in }}^{(i)}(n)}\left(\lambda_{\ell}^{(i)}-\gamma_{\ell}^{(i)}\right) \tilde{r}_{\ell}^{(i)}+\sum_{\ell \in \mathcal{L}_{\text {out }}(n), R x(\ell) \in \mathcal{D}_{i}}\left[\lambda_{\ell}^{(i)} \tilde{r}_{\ell}^{(i)}+\epsilon\left(\tilde{r}_{\ell}^{(i)}\right)^{2}\right]\right\} .
\end{aligned}
$$

The dual function is then obtained as

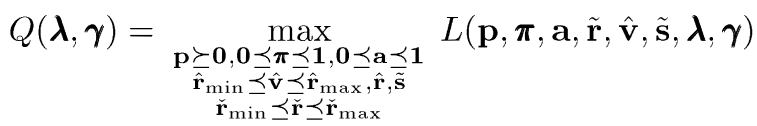$$
\text { s. to: (6), (16), and (19). }
$$

Note that $Q(\boldsymbol{\lambda}, \boldsymbol{\gamma})$ grows without bound if $\tilde{r}_{\ell}^{(i)}$ is sent to $-\infty$ provided $\gamma_{\ell}^{(i)}-\lambda_{\ell}^{(i)}<0$ for any $\ell \in \mathcal{L}_{\text {in }}^{(i)}(n), i \in \mathcal{J}_{n}, n \in \mathcal{N}$, since $L_{n}^{\text {high }}(\cdot)$ is linear in $\tilde{r}_{\ell}^{(i)}$, and (19) does not preclude $\tilde{r}_{\ell}^{(i)}$ from going to $-\infty$. Thus, the dual optimization problem is given by

$$
\begin{aligned}
& \min _{\boldsymbol{\lambda} \succeq \mathbf{0}, \boldsymbol{\gamma}} Q(\boldsymbol{\lambda}, \boldsymbol{\gamma}) \\
& \text { s. to: } \gamma_{\ell}^{(i)} \geq \lambda_{\ell}^{(i)},(\ell, i) \in \mathcal{A}_{n}, n \in \mathcal{N} .
\end{aligned}
$$

To solve the dual problem, (24) must be solved first. To this end, (24) can be decomposed into two subproblems. The first subproblem dealing with the MAC layer parameters is

$$
Q_{1}(\boldsymbol{\lambda}) \triangleq \max _{\substack{\mathbf{p} \succeq \mathbf{0}, \mathbf{0} \preceq \boldsymbol{\pi} \preceq \mathbf{1} \\ \mathbf{0} \preceq \mathbf{a} \preceq \mathbf{1}}} \sum_{n \in \mathcal{N}} L_{n}^{\mathrm{MAC}}\left(\mathbf{p}_{n}, \pi_{n}, \mathbf{a}_{n}, \boldsymbol{\lambda}_{n}, \overline{\boldsymbol{\lambda}}_{n}\right)
$$$$
\text { s. to: (6) and (16). }
$$

The second subproblem involving the higher-layer parameters is

$$
Q_{2}(\boldsymbol{\lambda}, \boldsymbol{\gamma}) \triangleq \max _{\substack{\hat{\mathbf{r}}_{\min } \preceq \hat{\mathbf{v}} \preceq \hat{\mathbf{r}}_{\max }, \hat{\mathbf{r}}, \tilde{\mathbf{s}} \\ \dot{\mathbf{r}}_{\text {mint }} \preceq \tilde{\mathbf{r}}_{n \in \mathcal{N}} \preceq \tilde{\mathbf{r}}_{\text {max }}}} \sum_{n}^{\mathrm{high}}\left(\tilde{\mathbf{s}}_{n}, \tilde{\mathbf{r}}_{n}, \hat{\mathbf{v}}_{n}, \tilde{\boldsymbol{\lambda}}_{n}, \boldsymbol{\gamma}_{n}\right)
$$

S. to: (19)

and $Q(\boldsymbol{\lambda}, \boldsymbol{\gamma})=Q_{1}(\boldsymbol{\lambda})+Q_{2}(\boldsymbol{\lambda}, \boldsymbol{\gamma})$. Next, each subproblems is solved in a distributed fashion.

\section{A. MAC Layer Subproblem}

To find the optimal primal variables associated with the random access MAC, one needs to solve (26), which is separable to individual nodes. The per-node optimization at node $n$ is

(P4)

$$
\begin{aligned}
& \max _{\substack{\mathbf{p}_{n} \succeq \mathbf{0 , 0} \preceq \mathbf{a}_{n} \preceq \mathbf{1} \\
0 \leq \pi_{n} \leq 1}} L_{n}^{\mathrm{MAC}}\left(\mathbf{p}_{n}, \pi_{n}, \mathbf{a}_{n}, \boldsymbol{\lambda}_{n}, \overline{\boldsymbol{\lambda}}_{n}\right) \\
& \text { s. to: (6) and (16). }
\end{aligned}
$$

The optimal persistence probabilities for node $n$ and its outgoing links are determined by solving the KKT optimality conditions for a given $\lambda$. Specifically, with $\Lambda_{n} \triangleq \sum_{\ell \in \mathcal{L}_{\text {out }}(n)} \lambda_{\ell}+$ $\sum_{\ell \in \mathcal{L}_{\text {from }}(n)} \lambda_{\ell}$, the optimal access probabilities are obtained as (cf. [15] and [31])

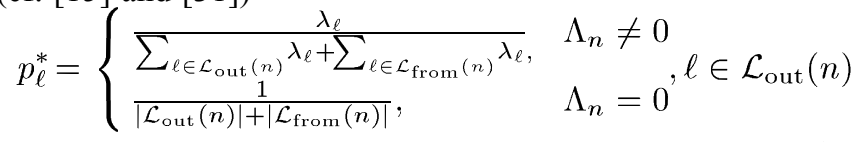

$$
\pi_{n}^{*}= \begin{cases}\frac{\sum_{\ell \in \mathcal{L}_{\text {out }}(n)} \lambda_{\ell}}{\frac{\sum_{\ell \in \mathcal{L}_{\text {out }}(n)} \lambda_{\ell}+\sum_{\ell \in \mathcal{L}_{\text {from }}(n)} \lambda_{\ell}}{\left|\mathcal{L}_{\text {out }}(n)\right|}} & \Lambda_{n} \neq 0 \\ \frac{\left|\mathcal{L}_{\text {out }}(n)\right|+\left|\mathcal{L}_{\text {from }}(n)\right|,}{} & \Lambda_{n}=0\end{cases}
$$

and the optimal $\left\{a_{(i)}^{(i) *}\right\}$ as

$$
a_{\ell}^{(i) *}=\left\{\begin{array}{ll}
\frac{\lambda_{\ell}^{(i)}}{\sum_{i \in \mathcal{J}_{n}} \lambda_{\ell}^{(i)}}, & \lambda_{\ell} \neq 0 \\
\frac{1}{\left|\mathcal{J}_{n}\right|}, & \lambda_{\ell}=0,
\end{array} \quad \ell \in \mathcal{L}_{\text {out }}(n), i \in \mathcal{J}_{n} .\right.
$$


Thus, to solve (P4) at the transmitter $n$ of link $\ell \in \mathcal{L}_{\text {out }}(n)$, node $n$ needs to collect only local dual variables $\left\{\lambda_{\ell}\right\}$ for $\ell \in$ $\mathcal{L}_{\text {from }}(n)$; all other dual variables are already local to node $n$. In Fig. 1 for example, node 4 needs to receive $\lambda_{\ell}$ associated with links $\{(3,4),(1,3),(2,3),(5,3),(6,3)\}$ from nodes $1,2,3,5$ and 6 through (at most two-hop) message passing. The MAC layer multipliers admit an intuitive pricing interpretation based on (29) and (30). Imagine a link $\ell$ with $\lambda_{\ell}>0$. If this link experiences frequent contention, the "contention price" $\lambda_{\ell}$ increases. Then, the link access probability $p_{\ell}^{*}$ increases and the transmission probability $\pi_{m}^{*}$ for the interfering nodes $m \in \mathcal{N}_{\text {to }}(\ell)$ decreases. This way the contention for link $\ell$ is relieved. Similarly, the dual prices $\lambda_{\ell}^{(i)}$ can also be interpreted as the price for the MAC resource for commodity $i$ in link $\ell$. The bandwidth fraction $a_{\ell}^{(i)}$ is allocated to commodity $i$ in proportion to the price in (31).

\section{B. Higher-Layer Subproblem}

Problem (27) represents an optimization task entailing network and transport layer variables. This problem can again be solved in a distributed fashion at the individual nodes. With $g_{n}^{(i)}\left(\tilde{\mathbf{s}}_{n}, \tilde{\mathbf{r}}_{n}, \hat{\mathbf{v}}_{n}\right)$ denoting the 1.h.s. of (19), the problem per node $n$ is

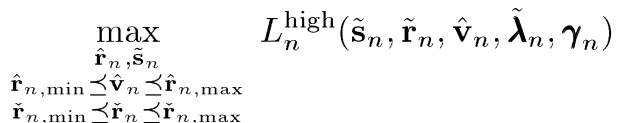

$$
\begin{aligned}
& \text { s. to: } g_{n}^{(i)}\left(\tilde{\mathbf{s}}_{n}, \tilde{\mathbf{r}}_{n}, \hat{\mathbf{v}}_{n}\right) \leq 0, \quad i \in \mathcal{J}_{n} \text {. }
\end{aligned}
$$

If $\boldsymbol{\mu}_{n} \triangleq\left[\mu_{n}^{(i)}\right]_{i \in \mathcal{J}_{n}}$ denote the Lagrange multipliers associated with constraints (33), the partial Lagrangian obtained by relaxing (33) is

$$
\begin{aligned}
\tilde{L}_{n}^{\text {high }}\left(\tilde{\mathbf{s}}_{n}, \tilde{\mathbf{r}}_{n}, \hat{\mathbf{v}}_{n}, \boldsymbol{\mu}_{n} ; \tilde{\boldsymbol{\lambda}}_{n}, \boldsymbol{\gamma}_{n}\right) & =L_{n}^{\mathrm{high}}\left(\tilde{\mathbf{s}}_{n}, \tilde{\mathbf{r}}_{n}, \hat{\mathbf{v}}_{n}, \tilde{\boldsymbol{\lambda}}_{n}, \boldsymbol{\gamma}_{n}\right) \\
& -\sum_{i \in \mathcal{J}_{n}} \mu_{n}^{(i)} g_{n}^{(i)}\left(\tilde{\mathbf{s}}_{n}, \tilde{\mathbf{r}}_{n}, \hat{\mathbf{v}}_{n}\right) .
\end{aligned}
$$

Assuming that Slater's conditions hold for (P5), the optimal primal and dual variables for (P5) must satisfy [3, Prop. 5.1.5]

$$
\begin{aligned}
& \left(\tilde{\mathbf{s}}_{n}^{*}, \tilde{\mathbf{r}}_{n}^{*}, \hat{\mathbf{v}}_{n}^{*}\right) \in \quad \arg \max \quad \tilde{L}_{n}^{\text {high }}\left(\tilde{\mathbf{s}}_{n}, \tilde{\mathbf{r}}_{n}, \hat{\mathbf{v}}_{n}, \boldsymbol{\mu}_{n}^{*} ; \tilde{\boldsymbol{\lambda}}_{n}, \boldsymbol{\gamma}_{n}\right) \\
& \widetilde{\mathbf{s}}_{n}, \hat{\mathbf{r}}_{n} \\
& \hat{\mathbf{r}}_{n, \min } \preceq \hat{\mathbf{v}}_{n} \preceq \hat{\mathbf{r}}_{n, \max } \\
& \check{\mathbf{r}}_{n, \min } \preceq \check{\mathbf{r}}_{n} \preceq \check{\mathbf{r}}_{n, \text { max }} \\
& \mu_{n}^{(i) *} g_{n}^{(i)}\left(\tilde{\mathbf{s}}_{n}^{*}, \tilde{\mathbf{r}}_{n}^{*}, \hat{\mathbf{v}}_{n}^{*}\right)=0, i \in \mathcal{J}_{n} \\
& g_{n}^{(i)}\left(\tilde{\mathbf{s}}_{n}^{*}, \tilde{\mathbf{r}}_{n}^{*}, \hat{\mathbf{v}}_{n}^{*}\right) \leq 0, i \in \mathcal{J}_{n} \\
& \mu_{n}^{(i) *} \geq 0, i \in \mathcal{J}_{n}
\end{aligned}
$$

where (36) is the complementary slackness condition for constraint (33), while (37) and (38) represent primal and dual feasibility, respectively.

Remarkably, the primal optimal variables $\left(\tilde{\mathbf{s}}_{n}^{*}, \tilde{\mathbf{r}}_{n}^{*}, \hat{\mathbf{v}}_{n}^{*}\right)$ satisfying (35)-(38) can be found very efficiently. The solution actually decouples into individual traffic commodities. For a source node $n \in \mathcal{S}_{i}$ of traffic commodity $i \in \mathcal{I}$, the corresponding primal optimal $\tilde{s}_{n}^{(i) *},\left\{\tilde{r}_{\ell}^{(i) *}\right\}_{\ell \in \mathcal{L}_{\mathrm{in}}^{(i)}(n)}$, $\left\{\tilde{r}_{\ell}^{(i) *}\right\}_{\ell \in \mathcal{L}_{\text {out }}(n), R x(\ell) \in \mathcal{D}_{i}}$, and $\left\{\tilde{v}_{\ell}^{(i) *}\right\}_{\ell \in \mathcal{L}_{\text {out }}^{(i)}(n)}$ can be obtained in closed form when $\beta_{n}^{(i)}=1$. When $\beta_{n}^{(i)}>1$, those optimal primal variables are expressed in closed form given the optimal dual variable $\mu_{n}^{(i) *}$, where $\mu_{n}^{(i) *}$ can be found as the unique root of a nonlinear equation, e.g., via a simple bisection search. Finally, for nodes $n \notin \mathcal{S}_{i}$ with $i \in \mathcal{I}$, the primal solutions $\left\{\tilde{r}_{\ell}^{(i) *}\right\}_{\ell \in \mathcal{L}_{\text {in }}^{(i)}(n)}$ and $\left\{\tilde{v}_{\ell}^{(i) *}\right\}_{\ell \in \mathcal{L}_{\text {out }}(n), R x(\ell) \in \mathcal{D}_{i}}$ are obtained in closed form regardless of the value of $\beta_{n}^{(i)}$.

Proposition 2: Upon defining $\Gamma_{n}^{(i)} \triangleq \sum_{\ell \in \mathcal{L}_{\text {in }}^{(i)}(n)}\left(\gamma_{\ell}^{(i)}-\right.$ $\left.\lambda_{\ell}^{(i)}\right), \tilde{z}_{\ell}^{(i) *} \triangleq \tilde{v}_{\ell}^{(i) *} \mathbb{1}_{\left\{R x(\ell) \notin \mathcal{D}_{i}\right\}}+\tilde{r}_{\ell}^{(i) *} \mathbb{1}_{\left\{R x(\ell) \in \mathcal{D}_{i}\right\}}$, and $\xi_{\ell}^{(i)} \triangleq$ $\gamma_{\ell}^{(i)} \mathbb{1}_{\left\{R x(\ell) \notin \mathcal{D}_{i}\right\}}+\lambda_{\ell}^{(i)} \mathbb{1}_{\left\{R x(\ell) \in \mathcal{D}_{i}\right\}}$, the globally optimal solution to (P5) for each $i \in \mathcal{J}_{n}$ is obtained as follows.

a) If $n \in \mathcal{S}_{i}$ and $\beta_{n}^{(i)}=1$, the outgoing log link rates are given by

$$
\tilde{z}_{\ell}^{(i) *}=\left[\frac{\left(w_{n}^{(i)}+\Gamma_{n}^{(i)}\right) \alpha_{\ell}^{(i)}(k)-\xi_{\ell}^{(i)}}{2 \epsilon}\right]_{\tilde{r}_{\ell, \min }^{(i)}}^{\tilde{r}_{\ell, \max }^{(i)}}, \quad \ell \in \mathcal{L}_{\mathrm{out}}(n)
$$

and the incoming log link rates by

$$
\tilde{r}_{\ell}^{(i) *}= \begin{cases}\log \left(\frac{\gamma_{\ell}^{(i)}-\lambda_{\ell}^{(i)}}{w_{n}^{(i)}+\Gamma_{n}^{(i)}} \prod_{\ell \in \mathcal{L}_{\text {out }}(n)}\left[\frac{e^{z_{\ell}^{(i) *}}}{\alpha_{\ell}^{(i)}(k)}\right]^{\alpha_{\ell}^{(i)}(k)}\right), \\ -\infty, \\ \gamma_{\ell}^{(i)}>\lambda_{\ell}^{(i)} \\ \gamma_{\ell}^{(i)}=\lambda_{\ell}^{(i)} \\ (40)\end{cases}
$$

The source log rate is also given by

$$
\tilde{s}_{n}^{(i) *}=\log \left(\frac{w_{n}^{(i)}}{w_{n}^{(i)}+\Gamma_{n}^{(i)}} \prod_{\ell \in \mathcal{L}_{\text {out }}(n)}\left[\frac{e^{\tilde{z}_{\ell}^{(i) *}}}{\alpha_{\ell}^{(i)}(k)}\right]^{\alpha_{\ell}^{(i)}(k)}\right) .
$$

b) If $n \in \mathcal{S}_{i}$ and $\beta_{n}^{(i)}>1$,

$\tilde{z}_{\ell}^{(i) *}\left(\mu_{n}^{(i) *}\right)=\left[\frac{\mu_{n}^{(i) *} \alpha_{\ell}^{(i)}(k)-\xi_{\ell}^{(i)}}{2 \epsilon}\right]_{\tilde{r}_{\ell, \min }^{(i)}}^{\tilde{r}_{\ell, \max }^{(i)}}, \quad \ell \in \mathcal{L}_{\text {out }}(n)$

$\tilde{r}_{\ell}^{(i) *}\left(\mu_{n}^{(i) *}\right)=\left\{\begin{aligned} \frac{\beta_{n}^{(i)}}{\beta_{n}^{(i)}-1} \log \left(\frac{w_{n}^{(i)}}{\mu_{n}^{(i) *}-\Gamma_{n}^{(i)}}\right) & +\log \left(\frac{\gamma_{\ell}^{(i)}-\lambda_{\ell}^{(i)}}{w_{n}^{(i)}}\right), \\ \gamma_{\ell}^{(i)} & >\lambda_{\ell}^{(i)}, \ell \in \mathcal{L}_{\text {in }}^{(i)}(n) \\ -\infty, & \gamma_{\ell}^{(i)}=\lambda_{\ell}^{(i)}, \ell \in \mathcal{L}_{\text {in }}^{(i)}(n)\end{aligned}\right.$

$$
\tilde{s}_{n}^{(i) *}\left(\mu_{n}^{(i) *}\right)=\frac{1}{\beta_{n}^{(i)}-1} \log \left(\frac{w_{n}^{(i)}}{\mu_{n}^{(i) *}-\Gamma_{n}^{(i)}}\right)
$$

where $\quad \mu_{n}^{(i) *} \quad$ satisfies $\quad g_{n}^{(i)}\left(\tilde{\mathbf{s}}_{n}^{*}\left(\mu_{n}^{(i) *}\right), \tilde{\mathbf{r}}_{n}^{*}\left(\mu_{n}^{(i) *}\right)\right.$, $\left.\tilde{\mathbf{v}}_{n}^{*}\left(\mu_{n}^{(i) *}\right)\right)=0$, which can be solved through a bisection search.

c) If $n \notin \mathcal{S}_{i}$,

$\tilde{z}_{\ell}^{(i) *}=\left[\frac{\Gamma_{n}^{(i)} \alpha_{\ell}^{(i)}(k)-\xi_{\ell}^{(i)}}{2 \epsilon}\right]_{\tilde{r}_{\ell, \min }^{(i)}}^{\tilde{r}_{\ell, \max }^{(i)}}, \quad \ell \in \mathcal{L}_{\text {out }}(n)$ 


$$
\begin{aligned}
& \int \log \left(\frac{\gamma_{\ell}^{(i)}-\lambda_{\ell}^{(i)}}{\Gamma_{n}^{(i)}} \prod_{\ell \in \mathcal{L}_{\text {out }}(n)}\left[\frac{e^{\tilde{z}_{\ell}^{(i) *}}}{\alpha_{\ell}^{(i)}(k)}\right]^{\alpha_{\ell}^{(i)}(k)}\right), \\
& \tilde{r}_{\ell}^{(i) *}= \begin{cases} & \gamma_{\ell}^{(i)}>\lambda_{\ell}^{(i)}, \Gamma_{n}^{(i)} \neq 0, \ell \in \mathcal{L}_{\text {in }}^{(i)}(n) \\
-\infty, & \gamma_{\ell}^{(i)}=\lambda_{\ell}^{(i)}, \Gamma_{n}^{(i)} \neq 0, \ell \in \mathcal{L}_{\text {in }}^{(i)}(n)\end{cases} \\
& \begin{array}{c}
\log \left(\frac{1}{\mid \mathcal{L}_{\text {in }}^{(i)}(n)} \prod_{\ell \in \mathcal{L}_{\text {out }}(n)}\left[\frac{e^{\bar{z}_{\ell}^{(i) *}}}{\alpha_{\ell}^{(i)}(k)}\right]^{\alpha_{\ell}^{(i)}(k)}\right), \\
\Gamma_{n}^{(i)}=0, \ell \in \mathcal{L}_{\text {in }}^{(i)}(n) .
\end{array}
\end{aligned}
$$

Proof: See Appendix A.

Referring to (9), one can see from (40) and (41) that for $n \in \mathcal{S}_{i}$ with $\beta_{n}^{(i)}=1$, the optimal $s_{n}^{(i) *}$ and $\left\{\tilde{r}_{\ell}^{(i) *}\right\}_{\ell \in \mathcal{L}^{(i)}(n)}$ divide a lower bound to the total outgoing flow in proportion to $w_{n}^{(i)}$ and $\left\{\left(\gamma_{\ell}^{(i)}-\lambda_{\ell}^{(i)}\right)\right\}$, respectively, in obedience to the flow conservation constraints. Thus, lower flow rates are assigned to links that experience higher MAC competition, and hence have larger values of $\lambda_{\ell}^{(i)}$. This indeed demonstrates the advantage of multipath routing to carry the traffic over links with lower contention and thus effect higher network utility. Similar observations can be made for the non-source nodes in (46). When $n \in \mathcal{S}_{i}$ and $\beta_{n}^{(i)}>1$, flow conservation is enforced by finding an appropriate $\mu_{n}^{(i) *}$ numerically.

It should be stressed that the solutions can be obtained in a distributed fashion, using only the dual variables local to - at most in the single-hop neighborhood of-node $n$, namely, $\left\{\left(\gamma_{\ell}^{(i)}-\lambda_{\ell}^{(i)}\right)\right\}_{\ell \in \mathcal{L}_{\text {in }}^{(i)}(n)},\left\{\gamma_{\ell}^{(i)}\right\}_{\ell \in \mathcal{L}_{\text {out }}^{(i)}(n)}$ and $\left\{\lambda_{\ell}^{(i)}\right\}_{\ell \in \mathcal{L}_{\text {out }}(n), R x(\ell) \in \mathcal{D}_{i}}$ for all $i \in \mathcal{J}_{n}$. For example, in Fig. 1, node 4 needs to receive $\left(\gamma_{\ell}^{(i)}-\lambda_{\ell}^{(i)}\right)$ for $(\ell, i) \in\{((3,4), 1),((3,4), 2)\}$ from node 3 .

\section{Dual Update}

To find the optimal dual variables, a subgradient projection method is employed to solve (25). From Danskin's theorem [3, p. 717], it follows that

$$
\begin{gathered}
\delta_{\ell}^{(i) \triangleq} \tilde{r}_{\ell}^{(i) *}-\log a_{\ell}^{(i) *}-\tilde{c}_{\ell}-\log p_{\ell}^{*}-\sum_{m \in \mathcal{N}_{\text {to }}(\ell)} \log \left(1-\pi_{m}^{*}\right) \\
\quad \in \partial_{\lambda_{\ell}^{(i)}} Q(\boldsymbol{\lambda}, \boldsymbol{\gamma}), \ell \in \mathcal{L}_{\text {out }}(n), i \in \mathcal{J}_{n}, n \in \mathcal{N}
\end{gathered}
$$

$$
\begin{array}{r}
\bar{\delta}_{\ell}^{(i) \triangleq} \tilde{v}_{\ell}^{(i) *}-\tilde{r}_{\ell}^{(i) *} \in \partial_{\gamma_{\ell}^{(i)}} Q(\boldsymbol{\lambda}, \boldsymbol{\gamma}), \\
\quad \ell \in \mathcal{L}_{\text {out }}^{(i)}(n), i \in \mathcal{J}_{n}, n \in \mathcal{N}
\end{array}
$$

where $\partial_{\lambda_{\ell}^{(i)}} Q(\boldsymbol{\lambda}, \boldsymbol{\gamma})$ and $\partial_{\gamma_{\ell}^{(i)}} Q(\boldsymbol{\lambda}, \boldsymbol{\gamma})$ are the subgradients of $Q(\cdot)$ at $(\boldsymbol{\lambda}, \boldsymbol{\gamma})$ w.r.t. $\lambda_{\ell}^{(i)}$ and $\gamma_{\ell}^{(i)}$, respectively. To ensure the dual variables belong to the feasible set of (25), projection is necessary. Upon defining $\mathcal{F} \triangleq\left\{(\boldsymbol{\lambda}, \boldsymbol{\gamma}) \mid \boldsymbol{\lambda} \succeq \mathbf{0}, \gamma_{\ell}^{(i)} \geq \lambda_{\ell}^{(i)},(\ell, i) \in \mathcal{A}_{n}, n \in \mathcal{N}\right\}$, $\mathcal{F}_{\ell}^{(i)} \triangleq\left\{\left(\lambda_{\ell}^{(i)}, \gamma_{\ell}^{(i)}\right) \mid \gamma_{\ell}^{(i)} \geq \lambda_{\ell}^{(i)}, \lambda_{\ell}^{(i)} \geq 0\right\}$ for $\ell \in \mathcal{L}_{\text {out }}^{(i)}(n)$, one can see that $\mathcal{F}$ is the Cartesian product of $\left\{\mathcal{F}_{\ell}^{(i)}\right\}(\ell, i) \in \mathcal{C}_{n}, n \in \mathcal{N}$ and $\left\{\lambda_{\ell}^{(i)} \mid \lambda_{\ell}^{(i)} \geq 0\right\}_{(\ell, i) \in \mathcal{B}_{n}, n \in \mathcal{N}}$. Thus, the subgradient projection step at time $t$ can be performed in a distributed fashion as

$$
\begin{aligned}
& \left(\lambda_{\ell}^{(i)}[t+1], \gamma_{\ell}^{(i)}[t+1]\right) \\
& =\mathcal{P}_{\mathcal{F}_{\ell}^{(i)}}\left\{\left(\lambda_{\ell}^{(i)}[t]+\sigma(t) \delta_{\ell}^{(i)}[t], \gamma_{\ell}^{(i)}[t]+\sigma(t) \bar{\delta}_{\ell}^{(i)}[t]\right)\right\}, \\
& \lambda_{\ell}^{(i)}[t+1] \\
& =\left[\lambda_{\ell}^{(i)}[t]+\sigma(t) \delta_{\ell}^{(i)}[t]\right]^{+},(\ell, i) \in \mathcal{B}_{n}, n \in \mathcal{N}
\end{aligned}
$$

where $[\cdot]^{+} \equiv \max \{0, \cdot\}$ and $\mathcal{P}_{\mathcal{F}_{\ell}^{(i)}}\{\cdot\}$ is the projection operation onto $\mathcal{F}_{\ell}^{(i)}$, which is given by

$$
\mathcal{P}_{\mathcal{F}_{\ell}^{(i)}}\{(\lambda, \gamma)\} \triangleq \begin{cases}(0, \gamma), & \lambda \leq 0, \gamma \geq 0 \\ \left(\frac{\lambda+\gamma}{2}, \frac{\lambda+\gamma}{2}\right), & \lambda+\gamma \geq 0, \lambda-\gamma \geq 0 \\ (0,0), & \gamma \leq 0, \lambda+\gamma \leq 0 \\ (\lambda, \gamma), & \text { otherwise. }\end{cases}
$$

Convergence of the iterates in (49) and (50) is asserted as follows.

Proposition 3: There exists a sequence of step sizes $\{\sigma(t)\}_{t=1}^{\infty}$ (e.g., $\left.\sigma(t)=\frac{1}{t}\right)$, for which the dual iterates $(\boldsymbol{\lambda}[t], \boldsymbol{\gamma}[t])$ converge to the dual optimal point $\left(\boldsymbol{\lambda}^{*}, \boldsymbol{\gamma}^{*}\right)$. If conditions c1) $\lambda_{\ell}^{*} \neq 0, \ell \in \mathcal{L}_{\text {out }}(n),\left|\mathcal{J}_{n}\right|>1$; c2) $\Lambda_{n}^{*} \neq 0$; and c3) $\Gamma_{n}^{(i) *} \neq 0, i \in \mathcal{J}_{n} \backslash \mathcal{I}_{n}$ hold for $n \in \mathcal{N}$, the primal variables recovered from the dual optimal variables through (29)-(31) and Prop. 2, are the optimal solution to (P3). ${ }^{1}$

Proof: (Sketch): The subgradient method with an appropriate sequence of step sizes converges to $\left(\boldsymbol{\lambda}^{*}, \boldsymbol{\gamma}^{*}\right)$ [27]. Problem (P3) is convex, and conditions c1)-c3) ensure that (P4) and (P5) have unique solutions at $\left(\boldsymbol{\lambda}^{*}, \boldsymbol{\gamma}^{*}\right)$. Thus, the primal variables obtained by maximizing the Lagrangian at the optimal dual variables are the optimal solution to the original problem [5, p. 248].

It is observed that the dual updates (49)-(50) can be performed in a distributed manner at each node. The overall distributed algorithm for solving (P3), which is equivalent to (P2), is listed in Table II. However, deciding when to stop the iteration by checking convergence may require central coordination. In practice, such a requirement may be circumvented by employing a global timer, which terminates the algorithm after a sufficient number of iterations.

With respect to the outer iterations in $k$ for successive convex approximation (cf. Table I), the algorithm in Table II comprises inner iterations. According to (10), updating $\left\{\alpha_{\ell}^{(i)}\right\}$ also requires only local information available at each node. Therefore, the overall optimization algorithm in Table I obtains a KKT point of the original problem (P1) in a distributed fashion.

Remark 1: The algorithms in the present work were derived with network synchronization tacitly assumed. However, synchronization is necessary only for the outer iterations (in

\footnotetext{
${ }^{1}$ Even without $\left.\left.\mathrm{c} 1\right)-\mathrm{c} 3\right)$, optimality is guaranteed by regularizing the objective in (P3) with the function $\bar{R}_{\epsilon}(\boldsymbol{\pi}, \mathbf{p}, \mathbf{a}, \hat{\mathbf{r}}) \triangleq-\epsilon \sum_{n \in \mathcal{N}}\left\{\pi_{n}^{2}+\right.$ $\left.\sum_{\ell \in \mathcal{L}_{\text {out }}(n)} p_{\ell}^{2}+\sum_{(\ell, i) \in \mathcal{B}_{n} \cup \mathcal{C}_{n}}\left(a_{\ell}^{(i)}\right)^{2}+\sum_{(\ell, i) \in \mathcal{C}_{n}} e^{\tilde{r}_{\ell}^{(i)}}\right\}$ which allows unique recovery of the primal variables. However, recovery of the primal variables may incur considerably higher complexity.
} 
TABLE II

Distributed Algorithm to Solve (P2) VIA (P3)

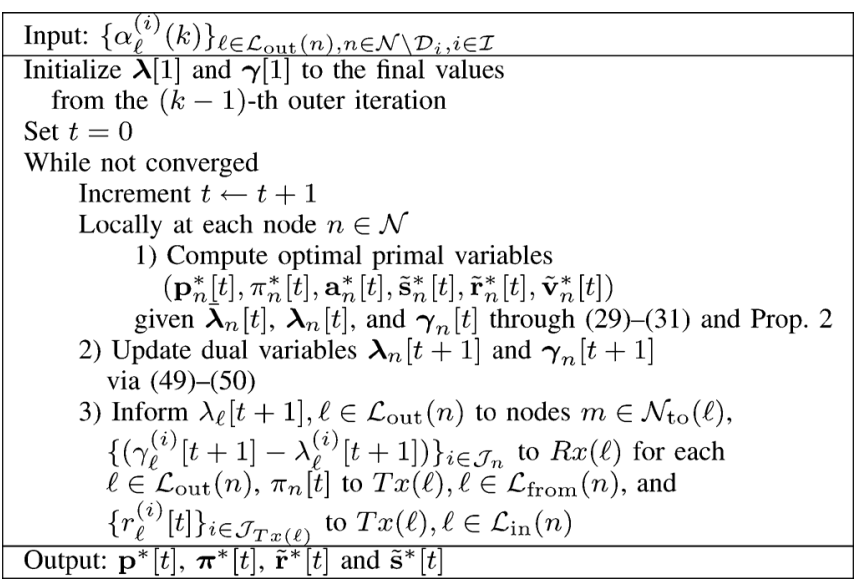

$k$ ) for the network to update the convex subproblem (P3). It can be shown that an asynchronous version of the algorithm in Table II can yield the optimal solution to (P3) under mild conditions [17].

\section{On-Line Implementation}

One may employ the proposed algorithm to obtain the optimal network parameters offline (either in a centralized or distributed fashion), and then deploy those parameters in the operational stage of the network. An alternative would be to perform the optimization online while the network is operational. Provided that queuing delay due to the transient behavior can be tolerated, the online alternative is advantageous in that slow variations in the network topology can be tracked.

Consider a slotted network whose time slots are aligned with the (inner) iteration of the proposed optimization algorithm. At each node $n \in \mathcal{N}$, network layer queues for each of the commodities in $\mathcal{J}_{n}$, as well as MAC layer queues for each of the outgoing links in $\mathcal{L}_{\text {out }}(n)$ are installed. At the beginning of each slot $t$, node $n$ collects the necessary dual variables from its neighborhood, and computes the corresponding primal variables by solving (P4) and (P5).

Subsequently, for $i \in \mathcal{I}_{n}$, the transport layer of node $n$ admits the commodity $i$ traffic from the higher layer into the commodity $i$ queue at the rate of $s_{n}^{(i) *}[t]$. Also, node $n$ receives the traffic from neighboring nodes through the incoming links. Among the incoming traffic, node $n$ delivers to the higher layer those commodities for which node $n$ is the intended destination. The rest is queued in the appropriate commodity queues at the network layer.

Then, packets in the network layer queue for commodity $i \in$ $\mathcal{J}_{n}$ are transferred to the MAC layer queue for link $\ell \in \mathcal{L}_{\text {out }}(n)$ at the rate of

$x_{\ell}^{(i) *}[t] \triangleq \begin{cases}\min _{\ell}\left\{v_{\ell}^{(i) *}[t], r_{\ell}^{(i) *}[t]\right\} & \text { if } \ell \in \mathcal{L}_{\text {out }}^{(i)}(n) \\ r_{\ell}^{(i) *}[t] & \text { if } \ell \in \mathcal{L}_{\text {out }}(n), R x(\ell) \in \mathcal{D}_{i}\end{cases}$

where the minimum is taken when $\ell \in \mathcal{L}_{\text {out }}^{(i)}(n)$ to ensure that the transmission rate does not exceed the reception rate $r_{\ell}^{(i) *}[t]$ dictated by the optimization algorithm.

To transmit the packets in the MAC layer queues, node $n$ tosses a coin and decides to transmit with optimal, per-slot persistence probability $\pi_{n}^{*}[t]$. Upon decision to transmit, an outgoing link $\ell$ is chosen randomly with probability $q_{\ell}^{*}[t]:=\frac{p_{\ell}^{*}[t]}{\sum_{\ell^{\prime} \in \mathcal{L}_{\text {out }}(n)} p_{\ell^{\prime}}^{*}[t]}$. For the chosen link $\ell^{*}$, the packets are transmitted at rate $c_{\ell^{*}}$. If the packets go through without collision, those successfully transmitted packets are removed from the MAC layer queue for link $\ell^{*}$. In view of (5), upon convergence of the optimization algorithm, the arrival rate per MAC queue is upper-bounded by the MAC achievable rate of the corresponding link. Thus, the MAC queues are stabilized. Likewise, network layer queues are also stabilized since the flow conservation constraints (19) are enforced, and the constraints (20) are met upon convergence.

\section{CONTEnTION GRAPH-BASED APPROACH}

To highlight the performance advantage of the proposed algorithm based on the precise probabilistic contention model, a benchmark is developed in this section. Specifically, the existing approach using the contention graph-based MAC model is considered for the joint congestion control, routing, and MAC optimization problem at hand. The model captures the MAC mechanism only approximately, resulting in performance degradation in practice.

A contention graph captures the interference relation of a given wireless network [7], [23]. Its vertices represent network links, and the undirected edges connecting the vertices indicate that the two links interfere with each other when both are active simultaneously. Thus, the set of vertices not sharing any edges can be active at the same time without causing interference. In the same token, in a fully connected subgraph (a clique) of the contention graph, only one vertex (link) is schedulable at a time. A maximal clique (a clique not inclusive in any other clique) of a contention graph is referred to as a contention region. In general, a centralized scheduler is necessary to schedule optimally for cross-layer objectives [6].

Schedulability constraints are usually approximated as clique feasibility constraints described in the following, although the latter do not necessarily imply the former. Since link $\ell$ with an average rate $r_{\ell}=\sum_{i \in \mathcal{I}_{n}} r_{\ell}^{(i)}$ utilizes an $\left(\frac{r_{\ell}}{c_{\ell}}\right)$-fraction of the air time, a necessary condition for a feasible schedule is given by

$$
\sum_{\ell \in \mathcal{C}_{j}} \frac{r_{\ell}}{c_{\ell}} \leq 1, \quad j=1,2, \ldots, N_{C}
$$

where $\mathcal{C}_{j}$ denotes the $j$ th set among the $N_{C}$ contention regions. In order to derive a distributed online algorithm, the approach in [13] is taken. Specifically, (53) is augmented to the optimization objective through penalty terms as

$$
\begin{aligned}
& \text { (P6) } \max _{\mathbf{r}_{\min } \preceq \mathbf{r} \preceq \mathbf{r}_{\max }} \sum_{i \in \mathcal{I} \succeq \mathbf{0}} \sum_{n \in \mathcal{S}_{i}} w_{n}^{(i)} U_{n}^{(i)}\left(s_{n}^{(i)}\right) \\
& -\rho \sum_{j=1}^{N_{C}} \int_{0}^{\sum_{\ell \in \mathcal{C}_{j}}{ }^{\frac{r_{\ell}}{c_{\ell}}}} \pi_{j}(v) d v-R_{\epsilon}^{\prime}(\mathbf{r}) \\
& \text { s. to: } \sum_{\ell \in \mathcal{L}_{\text {out }}(n)} r_{\ell}^{(i)} \geq \sum_{\ell \in \mathcal{L}_{\text {in }}^{(i)}(n)} r_{\ell}^{(i)}+s_{n}^{(i)} \mathbb{1}_{\left\{n \in \mathcal{S}_{i}\right\}} \text {, } \\
& n \in \mathcal{N} \backslash \mathcal{D}_{i}, i \in \mathcal{I}
\end{aligned}
$$


where $R_{\epsilon}^{\prime}(\mathbf{r}) \triangleq \epsilon \sum_{n \in \mathcal{N} \backslash \mathcal{D}} \sum_{\ell \in \mathcal{L}_{\text {out }}(n)} \sum_{i \in \mathcal{I}_{n}}\left(r_{\ell}^{(i)}\right)^{2}$ is the regularization term ensuring uniqueness of the solution, $\pi_{j}(\cdot)$ the penalty function to enforce (53) for the $j$ th contention region, and $\rho$ the parameter for tuning the severity of the penalty. It is assumed that $\pi_{j}(\cdot)$ are continuous, nondecreasing and nonnegative, leading to a convex formulation (P6). Thus, upon applying the dual decomposition method and relaxing the constraints (55) using the Lagrange multipliers $\left\{\lambda_{n}^{(i)}\right\}$, an iterative algorithm can be derived.

Let $t$ be the iteration index. Given the Lagrange multipliers at iteration $t$, the source rates can be obtained as

$$
\begin{aligned}
& s_{n}^{(i) *}[t]=\arg \max _{s_{n}^{(i)} \geq 0}\left\{w_{n}^{(i)} U_{n}^{(i)}\left(s_{n}^{(i)}\right)-\lambda_{n}^{(i)}[t] s_{n}^{(i)}\right\}, \\
& n \in \mathcal{S}_{i}, i \in \mathcal{I} .
\end{aligned}
$$

In principle, the link rates $\left\{r_{\ell}^{(i) *}[t]\right\}$ at iteration $t$ can be obtained using the gradient projection method involving subiterations indexed by $k^{\prime}$ :

$$
\begin{aligned}
& r_{\ell}^{(i)}\left[t, k^{\prime}+1\right] \\
& =\left[r_{\ell}^{(i)}\left[t, k^{\prime}\right]-\frac{\rho \sigma}{c_{\ell}} \sum_{j: \ell \in \mathcal{C}_{j}} \pi_{j}\left(\sum_{\ell \in \mathcal{C}_{j}} \frac{r_{\ell}\left[t, k^{\prime}\right]}{c_{\ell}}\right)\right. \\
& \left.\quad-\sigma\left(\lambda_{R x(\ell)}^{(i)}[t]-\lambda_{T x(\ell)}^{(i)}[t]+2 \epsilon r_{\ell}^{(i)}\left[t, k^{\prime}\right]\right)\right]_{r_{\ell, \min }^{(i)}}^{r_{\ell, \max }^{(i)}}, \\
& \quad \ell \in \mathcal{L}_{\text {out }}^{(i)}(n), i \in \mathcal{J}_{n}, n \in \mathcal{N} \quad(57)
\end{aligned}
$$

where $\sigma$ denotes the step size. To avoid explicit exchange of information among the contention regions, the ratio $\frac{r_{\ell}}{c_{\ell}}$ is interpreted as the access probability of link $\ell$, and the update in (57) is performed online as follows [13]. If $T x(\ell)$ has a packet to send, it transmits with probability $p_{\ell}\left[t, k^{\prime}\right]=\min \left\{1, \frac{r_{\ell}\left[t, k^{\prime}\right]}{c_{\ell}}\right\}$. (When multiple outgoing links of a node have packets to send, one of the links is randomly picked.) If collision occurs, the rate $r_{\ell}\left[t, k^{\prime}\right]$ is decreased by $\frac{\sigma \rho}{c \ell}$. Also, $r_{\ell}^{(i)}\left[t, k^{\prime}\right]$ is decreased by $\sigma\left(\lambda_{R x(\ell)}^{(i)}[t]-\lambda_{T x(\ell)}^{(i)}[t]+2 \epsilon r_{\ell}^{(i)}\left[t, k^{\prime}\right]\right)$ per subiteration $k^{\prime}$.

Finally, the dual variables are updated as

$\lambda_{n}^{(i)}[t+1]=$

$$
\left[\lambda_{n}^{(i)}[t]-\sigma\left(\sum_{\ell \in \mathcal{L}_{\text {out }}(n)} r_{\ell}^{(i) *}[t]-\sum_{\ell \in \mathcal{L}_{\in}^{(i)}(n)} r_{\ell}^{(i) *}[t]-s_{n}^{(i) *}[t]\right)\right]^{+},
$$

\section{NUMERICAL TESTS}

The performance of the proposed algorithm is assessed via numerical tests. Wireless networks with bidirectional links are considered, where two nodes can hear (and interfere) each other if their distance is less than a threshold distance $d_{c}$. The subgradient algorithm with constant step size $\sigma(t)=10^{-4}, \forall t$ is employed. Parameter values $\epsilon=10^{-4}, c_{\ell}=10, r_{\ell \text { min }}^{(i)}=10^{-3}$, $r_{\ell, \max }^{(i)}=10$, and $w_{n}^{(i)}=1, \forall \ell, n, i$, are used.

\section{A. Small Network Example}

As the first example, the network topology in Fig. 1 is considered with $d_{c}=0.5$. As discussed in Section II, $\mathcal{I}=\{1,2\}$,

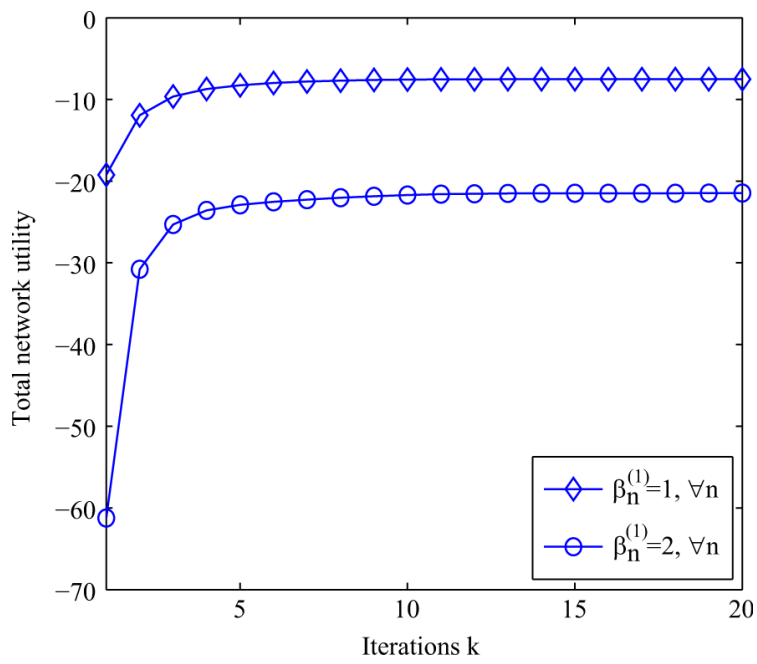

Fig. 2. Convergence of outer iterations.

with the diamond and the square representing the destination nodes of commodities 1 and 2, respectively. All nodes generate both commodities, except the destination nodes, which generate single commodities. Fig. 2 depicts the convergence behavior of the network utility as the outer iteration (indexed by $k$ ) progresses. It is observed that the objectives increase monotonically until convergence. The converged link flow rates and node persistence probabilities are illustrated in Fig. 1, where the thickness of the links is roughly proportional to the associated flow rates, and the radius of the circles to the persistence probabilities.

Fig. 3 shows the evolution of the inner iterations (indexed by t). Fig. 3(a) depicts the evolution of the primal objectives (total network utilities) and the dual objectives. Different groups of curves correspond to different outer iteration index $k$. It should be noted that the primal and the dual objectives shown in Fig. 3(a) correspond to those of the convex subproblem (P3). Since (P3) is convex, there is no duality gap. As depicted in Fig. 3(b), the number of inner iterations required for convergence is reduced rapidly as the outer iteration in $k$ progresses, since the solution from the previous outer iteration is used as the starting point. The duality gap being less than $10^{-2}$ was used as the convergence criterion. The evolution of the dual variables $\left\{\lambda_{\ell}^{(i)}\right\}$ is plotted for $k=4$ in Fig. 3(c).

\section{B. Larger Network Example}

For a larger network example, a network with 15 nodes randomly placed in a unit square is considered with $d_{c}=0.35$. To see the performance advantage of the proposed design, two existing alternatives are tested. The first alternative is the joint congestion control and MAC optimization method proposed in [31], which does not jointly optimize routing. The second approach is the contention graph-based benchmark derived in Section V.

1) Comparison with the method in [31]: This algorithm assumes that the routes are prespecified and performs optimization of end-to-end throughput and access probabilities. Minimum hop-count routes were used. The average optimized network utilities obtained by averaging 10 random topologies for various fairness criteria are listed in Table III. It is observed that our algorithm noticeably outperforms the scheme that does not perform joint route optimization. 


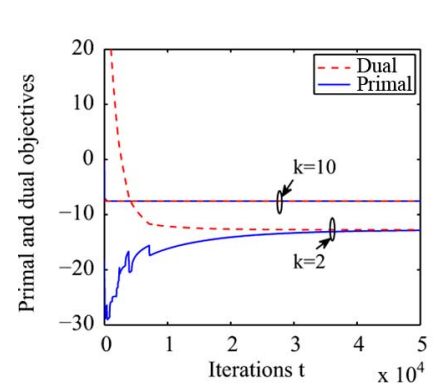

(a)

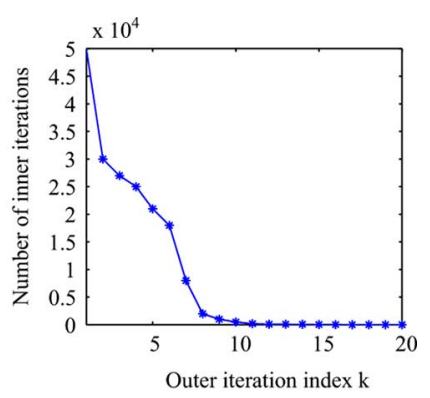

(b)

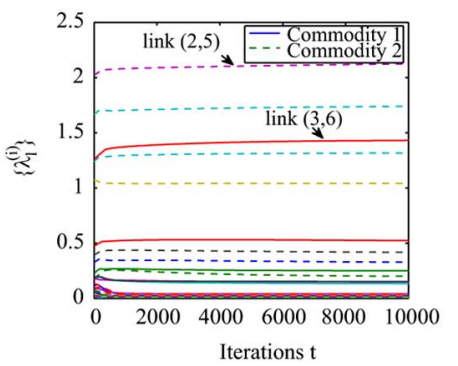

(c)

Fig. 3. Convergence of inner iterations (a) Primal and dual objectives over different outer iterations (b) Number of inner iterations as the outer iteration progresses (c) Dual variables $\left\{\lambda_{\ell}^{(i)}\right\}$ for $k=4$.

TABLE III

Optimized Network Utilities AVERAged OVER TEN RANDOM NETWORK TOPOLOGIES

\begin{tabular}{|c|c|c|c|}
\hline Algorithm & $\beta_{n}^{(1)}=1, \forall n$ & $\beta_{n}^{(1)}=2, \forall n$ & $\beta_{n}^{(1)}=5, \forall n$ \\
\hline proposed method & 0.76 & -3.32 & -0.61 \\
\hline method in [31] & 0.14 & -4.1 & -1.8 \\
\hline contention graph & 0.51 & -3.69 & -0.85 \\
\hline
\end{tabular}

An instance of the simulated topologies is shown in Fig. 4, which entails 52 directional links. Four source nodes marked by squares inject single-commodity traffic destined to the node marked by the diamond. Table IV lists the maximized total network utilities and the optimal source rates obtained from both algorithms using the proportional fairness $\left(\beta_{n}^{(1)}=1\right)$, and the harmonic-mean fairness $\left(\beta_{n}^{(1)}=2\right)$ utilities. It is seen that the proposed algorithm achieves higher throughput than that of [31] under the same fairness criteria. The routes and the converged link flow rates for the proportional fairness case are depicted in Fig. 4(a), where again the thickness of the links is approximately proportional to the associated flow rates. It can be seen that the minimum hop count-based routing generates intense contention near the destination, while our algorithm intelligently selects the routing paths so as to avoid interference-prone areas of the network.

2) Comparison with the contention graph-based method: Simulations are performed to show the benefits of probabilistic method adopted in this paper compared to the contention graph-based method derived in Section V. The value of $\rho$ was set to 0.01 . The average network utilities obtained from 10 different random network topologies are shown in Table IV. It can be seen that the crude approximation of the contention graph model degrades the cross-layer performance.

For the particular network topology in Fig. 4, the utilities and source rates are again presented in Table III for proportional and

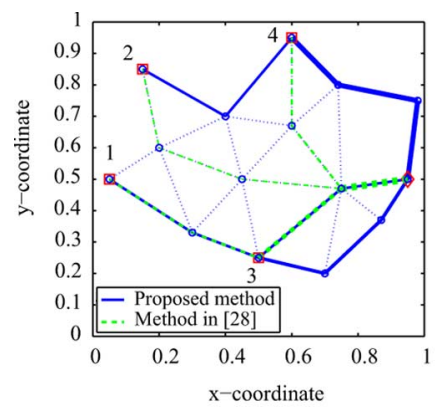

(a)

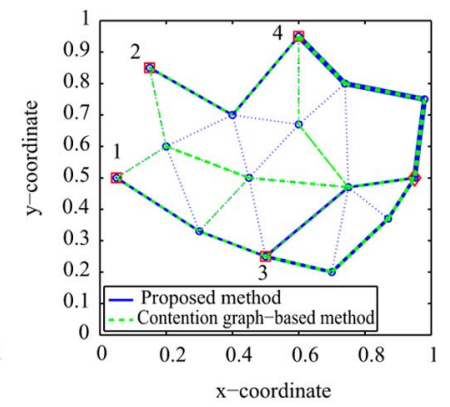

(b)
Fig. 4. An instance of the experimented network topologies with the optimized flow rates. (a) Comparison with the method in [31]. (b) Comparison with the contention graph-based method.

harmonic-mean fairness objectives. The optimized routes and their corresponding flow rates are also depicted in Fig. 4(b). It can be seen that the contention graph-based scheme tends to use more paths than the proposed scheme, which may increase the number of collisions in the network.

3) Robustness to initial points: To gauge the sensitivity of the proposed method to the starting point of the iterative algorithm, the optimal proportional fairness objective values obtained from 1,000 different randomly selected feasible initial points for the network topology in Fig. 4 are depicted in Fig. 5 (for $\epsilon=10^{-3}$ ). Although the proposed algorithm is designed to find the locally optimal solutions, it is seen that the obtained solutions coincide with the global optimum quite often (around $80 \%$ of the trials).

\section{Online Network Control}

Simulated tests are also performed to validate the online implementation described in Section IV-D. The network topology in Fig. 1 is used with fully backlogged transport layer queues. Node 1 maintains two network layer queues for commodities 1 and 2, and two MAC layer queues for links $(1,2)$ and $(1,3)$. For each outer iteration $k, 10^{5}$ inner iterations are used. Fig. 6 depicts the evolution of the network-layer and the MAC-layer queue lengths at node 1 . It can be seen that the queue lengths eventually drop to zero, and the network is stabilized. Similar trends are observed for the queues in the remaining nodes.

\section{CONCLUSION}

Cross-layer optimization of flow control, multipath routing and Aloha random access control was investigated for wireless multi-commodity multihop networks using the NUM framework. Unlike the case where routes are fixed a priori, the joint routing optimization problem turns out to be inherently nonconvex. To mitigate this impasse, a successive convex approximation approach was adopted, which yields a KKT optimal point of the problem. By introducing auxiliary variables and exploiting the problem structure, a scalable distributed algorithm was developed, which requires very low computational complexity per node. An online implementation of the proposed algorithm was also proposed. The numerical tests verified the efficacy of our design. 
TABLE IV

OptIMIZED NeTWORK UTILITIES AND SOURCE RATES

\begin{tabular}{|c|c|c|c|c|c|}
\hline Algorithm & Network Utility & $s_{1}^{(1) *}$ & $s_{2}^{(1) *}$ & $s_{3}^{(1) *}$ & $s_{4}^{(1) *}$ \\
\hline proposed $\left(\beta_{n}^{(1)}=1, \forall n\right)$ & 0.67 & 1.08 & 1.07 & 1.28 & 1.29 \\
\hline method in [31] $\left(\beta_{n}^{(1)}=1, \forall n\right)$ & -0.97 & 0.87 & 0.69 & 0.88 & 0.70 \\
\hline contention graph-based $\left(\beta_{n}^{(1)}=1, \forall n\right)$ & -0.2 & 0.77 & 0.77 & 1.18 & 1.15 \\
\hline \hline proposed $\left(\beta_{n}^{(1)}=2, \forall n\right)$ & -3.38 & 1.13 & 1.13 & 1.23 & 1.23 \\
\hline method in [31] $\left(\beta_{n}^{(1)}=2, \forall n\right)$ & -5.11 & 0.82 & 0.74 & 0.82 & 0.74 \\
\hline contention graph-based $\left(\beta_{n}^{(1)}=2, \forall n\right)$ & -4.1 & 0.87 & 0.88 & 1.1 & 1.08 \\
\hline
\end{tabular}

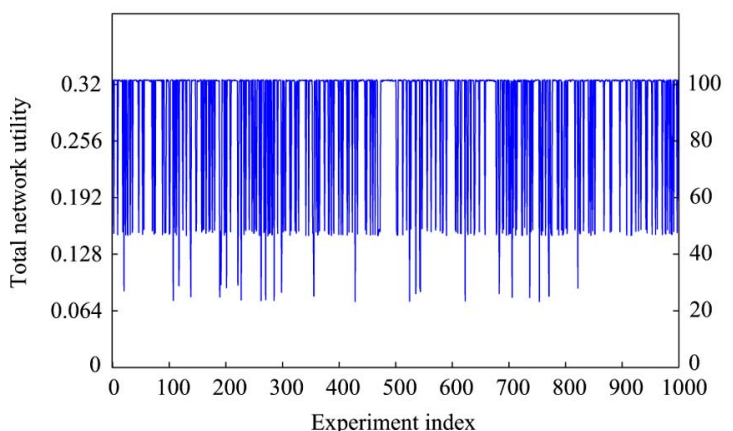

Fig. 5. Network utility obtained from random initial points when $\beta_{n}^{(1)}=1, \forall n$.

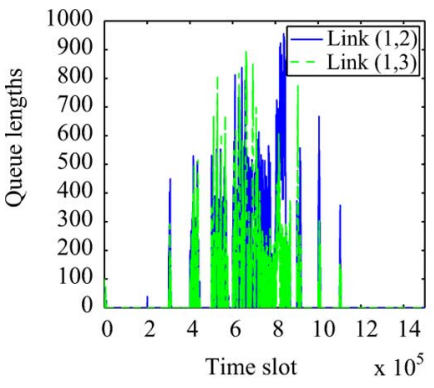

(a)

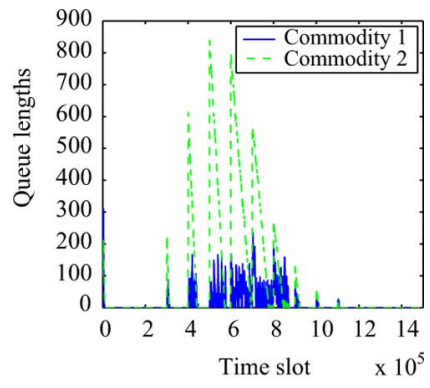

(b)
Fig. 6. Evolution of queue lengths at node 1. (a) MAC layer queues. (b) Network layer queues.

APPENDIX A

PROOF OF PROPOSITION 2

Start by writing $\tilde{L}_{n}^{\text {high }}\left(\tilde{\mathbf{s}}_{n}, \tilde{\mathbf{r}}_{n}, \hat{\mathbf{v}}_{n}, \boldsymbol{\mu}_{n} ; \tilde{\boldsymbol{\lambda}}_{n}, \boldsymbol{\gamma}_{n}\right)$ as

$$
\begin{aligned}
& \tilde{L}_{n}^{\mathrm{high}}(\cdot)=\sum_{i \in \mathcal{J}_{n}}\left\{_{\ell \in \mathcal{L}_{\text {out }}^{(i)}(n)}\left[\left(-\gamma_{\ell}^{(i)}+\mu_{n}^{(i)} \alpha_{\ell}^{(i)}\right) \tilde{v}_{\ell}^{(i)}-\epsilon\left(\tilde{v}_{\ell}^{(i)}\right)^{2}\right]\right. \\
& +\sum_{\substack{\ell \in \mathcal{L}_{\text {out }}(n) \\
R x(\ell) \in \mathcal{D}_{i}}}\left[\left(-\lambda_{\ell}^{(i)}+\alpha_{\ell}^{(i)} \mu_{n}^{(i)}\right) \tilde{r}_{\ell}^{(i)}-\epsilon\left(\tilde{r}_{\ell}^{(i)}\right)^{2}\right] \\
& +w_{n}^{(i)} \tilde{U}_{n}^{(i)}\left(\tilde{s}_{n}^{(i)}\right) \mathbb{1}_{\left\{n \in \mathcal{S}_{i}\right\}}+\sum_{\ell \in \mathcal{L}_{\text {in }}^{(i)}(n)}\left(\gamma_{\ell}^{(i)}-\lambda_{\ell}^{(i)}\right) \tilde{r}_{\ell}^{(i)} \\
& -\mu_{n}^{(i)} \log \left(e^{\tilde{s}_{n}^{(i)}} \mathbb{1}_{\left\{n \in \mathcal{S}_{i}\right\}}+\sum_{\ell \in \mathcal{L}_{\mathrm{in}}^{(i)}(n)} e^{\tilde{r}_{\ell}^{(i)}}\right) \\
& \left.-\mu_{n}^{(i)} \sum_{\ell \in \mathcal{L}_{\text {out }}(n)} \alpha_{\ell}^{(i)}(k) \log \alpha_{\ell}^{(i)}(k)\right\} \text {. }
\end{aligned}
$$

The maximization in (35) is separable in $i \in \mathcal{J}_{n}$, and decomposable to maximizations over $\left\{\tilde{v}_{\ell}^{(i)}\right\}_{\ell \in \mathcal{L}_{\text {out }}^{(i)}(n)}$,

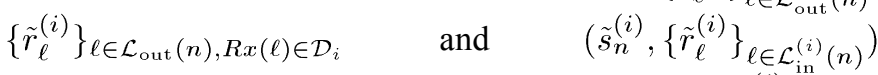
for each $i$. The subproblems pertaining to $\left\{\tilde{v}_{\ell}^{(i)}\right\}$ and $\left\{\tilde{r}_{\ell}^{(i)}\right\}_{\ell \in \mathcal{L}_{\text {out }}(n), R x(\ell) \in \mathcal{D}_{i}}$ are further decomposable per $\ell$ as

$$
\begin{array}{r}
\tilde{v}_{\ell}^{(i)}=\underset{\substack{\tilde{r}_{\ell, \min }^{(i)} \leq \tilde{v}_{\ell}^{(i)} \leq \tilde{r}_{\ell, \max }^{(i)} \\
\arg \max }}{ }\left\{\left(-\gamma_{\ell}^{(i)}+\mu_{n}^{(i) *} \alpha_{\ell}^{(i)}(k)\right) \tilde{v}_{\ell}^{(i)}-\epsilon\left(\tilde{v}_{\ell}^{(i)}\right)^{2}\right\}, \\
\ell \in \mathcal{L}_{\text {out }}^{(i)}(n)
\end{array}
$$

$\tilde{r}_{\ell}^{(i)}=\underset{\tilde{r}_{\ell, \min }^{(i)} \leq \tilde{r}_{\ell}^{(i)} \leq \tilde{r}_{\ell, \max }^{(i)}}{\arg \max }\left\{\left(-\lambda_{\ell}^{(i)}+\mu_{n}^{(i) *} \alpha_{\ell}^{(i)}(k)\right) \tilde{r}_{\ell}^{(i)}-\epsilon\left(\tilde{r}_{\ell}^{(i)}\right)^{2}\right\}$,

$$
\ell \in \mathcal{L}_{\text {out }}(n), R x(\ell) \in \mathcal{D}_{i},
$$

respectively. Since (60) and (61) are quadratic and concave in $\tilde{v}_{\ell}^{(i)}$ and $\tilde{r}_{\ell}^{(i)}$, the corresponding optimal values are uniquely determined for a given $\mu_{n}^{(i) *}$ as (42). The optimal values $\left(\tilde{s}_{n}^{(i) *},\left\{\tilde{r}_{\ell}^{(i) *}\right\}_{\ell \in \mathcal{L}_{\text {in }}^{(i)}(n)}\right)$ must maximize

$$
\begin{aligned}
w_{n}^{(i)} \tilde{U}_{n}^{(i)}\left(\tilde{s}_{n}^{(i)}\right) \mathbb{1}_{\left\{n \in \mathcal{S}_{i}\right\}}+\sum_{\ell \in \mathcal{L}_{\mathrm{in}}^{(i)}(n)}\left(\gamma_{\ell}^{(i)}-\lambda_{\ell}^{(i)}\right) \tilde{r}_{\ell}^{(i)} \\
-\mu_{n}^{(i)} \log \left(e^{\tilde{s}_{n}^{(i)}} \mathbb{1}_{\left\{n \in \mathcal{S}_{i}\right\}}+\sum_{\ell \in \mathcal{L}_{\mathrm{in}}^{(i)}(n)} e^{\tilde{r}_{\ell}^{(i)}}\right)
\end{aligned}
$$

which we denote as $f_{n}^{(i)}\left(\tilde{s}_{n}^{(i)},\left\{\tilde{r}_{\ell}^{(i)}\right\}\right)$.

Consider first the case where $n \in \mathcal{S}_{i}$. The derivative of $f_{n}^{(i)}(\cdot)$ w.r.t. $\tilde{s}_{n}^{(i)}$ is

$$
\begin{aligned}
\frac{\partial f_{n}^{(i)}\left(\tilde{s}_{n}^{(i)},\left\{\tilde{r}_{\ell}^{(i)}\right\}\right)}{\partial \tilde{s}_{n}^{(i)}}=w_{n}^{(i)} \frac{d \tilde{U}_{n}^{(i)}\left(\tilde{s}_{n}^{(i)}\right)}{d \tilde{s}_{n}^{(i)}} & \\
& -\frac{\mu_{n}^{(i) *} e^{\tilde{s}_{n}^{(i)}}}{e^{\tilde{s}_{n}^{(i)}}+\sum_{\ell \in \mathcal{L}_{\epsilon}^{(i)}(n)} e^{\tilde{r}_{\ell}^{(i)}}}
\end{aligned}
$$

If $\mu_{n}^{(i) *}=0$, the derivative is positive since $\frac{d \tilde{U}_{n}^{(i)}\left(\tilde{s}_{n}^{(i)}\right)}{d \tilde{s}_{n}^{(i)}}=$

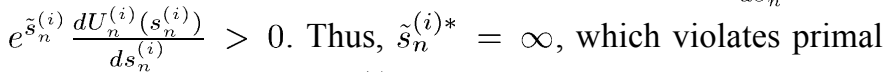
feasibility (37). Hence, $\mu_{n}^{(i) *}>0$ must hold, and from (36) we have

$$
g_{n}^{(i)}\left(\tilde{\mathbf{s}}_{n}^{*}, \tilde{\mathbf{r}}_{n}^{*}, \hat{\mathbf{v}}_{n}^{*}\right)=0
$$


Since $\mu_{n}^{(i) *}>0$ and $\tilde{s}_{n}^{(i)}$ is unconstrained, (63) equals to zero at the optimum, which gives

$$
w_{n}^{(i)} e^{-\left(\beta_{n}^{(i)}-1\right) \tilde{s}_{n}^{(i) *}}-\frac{\mu_{n}^{(i) *} e^{\tilde{s}_{n}^{(i) *}}}{e^{\tilde{s}_{n}^{(i) *}}+\sum_{\ell \in \mathcal{L}_{\mathrm{in}}^{(i)}(n)} e^{\tilde{\tilde{r}}_{\ell}^{(i) *}}}=0
$$

for $\beta_{n}^{(i)} \geq 1$. Now, the derivative of $f_{n}^{(i)}(\cdot)$ w.r.t. $\tilde{r}_{\ell}^{(i)}$ for $\ell \in$ $\mathcal{L}_{\text {in }}^{(i)}(n)$ is given by

$$
\begin{aligned}
\frac{\partial f_{n}^{(i)}\left(\tilde{s}_{n}^{(i)},\left\{\tilde{r}_{\ell}^{(i)}\right\}\right)}{\partial \tilde{r}_{\ell}^{(i)}} & =\gamma_{\ell}^{(i)}-\lambda_{\ell}^{(i)} \\
& -\frac{\mu_{n}^{(i) *} e^{\tilde{r}_{\ell}^{(i)}}}{e^{\tilde{s}_{n}^{(i)}}+\sum_{\ell \in \mathcal{L}_{\mathrm{in}}^{(i)}(n)} e^{\tilde{r}_{\ell}^{(i)}}}, \ell \in \mathcal{L}_{\mathrm{in}}^{(i)}(n) .
\end{aligned}
$$

If $\gamma_{\ell}^{(i)}=\lambda_{\ell}^{(i)}$, then the derivative is negative, and $\tilde{r}_{\ell}^{(i) *}=$ $-\infty$. For the rest of $\ell \in \mathcal{L}_{\text {in }}^{(i)}(n)$, where $\gamma_{\ell}^{(i)}>\lambda_{\ell}^{(i)}, \tilde{r}_{\ell}^{(i) *}$ is chosen so that (66) is equal to zero:

$$
\gamma_{\ell}^{(i)}-\lambda_{\ell}^{(i)}-\frac{\mu_{n}^{(i) *} e^{\tilde{r}_{\ell}^{(i) *}}}{e^{\tilde{s}_{n}^{(i) *}}+\sum_{\ell \in \mathcal{L}_{\epsilon}^{(i)}(n)} e^{\tilde{r}_{\ell}^{(i) *}}}=0, \ell \in \mathcal{L}_{\in}^{(i)}(n) .
$$

From (65) and (67), it follows that

$$
\tilde{r}_{\ell}^{(i) *}=\beta_{n}^{(i)} \tilde{s}_{n}^{(i) *}+\log \left(\frac{\gamma_{\ell}^{(i)}-\lambda_{\ell}^{(i)}}{w_{n}^{(i)}}\right), \ell \in \mathcal{L}_{\text {in }}^{(i)}(n) .
$$

Substituting (68) into (65) yields

$$
w_{n}^{(i)}+\left(\Gamma_{n}^{(i)}-\mu_{n}^{(i) *}\right) e^{\left(\beta_{n}^{(i)}-1\right) \tilde{s}_{n}^{(i) *}}=0 .
$$

When $\beta_{n}^{(i)}=1,(69)$ yields the optimal Lagrange multiplier $\mu_{n}^{(i) *}$ in closed form as

$$
\mu_{n}^{(i) *}=w_{n}^{(i)}+\Gamma_{n}^{(i)}
$$

and (39) is obtained by replacing $\mu_{n}^{(i) *}$ in (42) by (70). Equation (41) is derived by eliminating $\left\{\tilde{r}_{\ell}^{(i) *}\right\}_{\ell \in \mathcal{L}_{\text {in }}^{(i)}(n)}$ in (64) through (65) and solving for $\tilde{s}_{n}^{(i) *}$. Substituting this $\tilde{s}_{n}^{(i) *}$ in (68) yields (40).

When $\beta_{n}^{(i)}>1$, (44) is obtained by solving for $\tilde{s}_{n}^{(i) *}$ in (69), where it is noted that $\mu_{n}^{(i) *}>\Gamma_{n}^{(i)}$. Again, substituting this $\tilde{s}_{n}^{(i) *}$ into (68) yields (43). To obtain $\mu_{n}^{(i) *},(42)-(44)$ are plugged into (64), which yields the condition $h_{n}^{(i)}\left(\mu_{n}^{(i) *}\right)=0$, where

$$
\begin{aligned}
& h_{n}^{(i)}(x) \triangleq \\
& \sum_{\ell \in \mathcal{L}_{\text {out }}(n)} \alpha_{\ell}^{(i)}(k)\left\{\left[\frac{\alpha_{\ell}^{(i)}(k) x-\xi_{\ell}^{(i)}}{2 \epsilon}\right]_{\tilde{r}_{\ell, \min }^{(i)}}^{\tilde{r}_{\ell(i)}^{(i)}}-\log \alpha_{\ell}^{(i)}(k-1)\right\} \\
& +\log \left(\frac{w_{n}^{(i)}}{x}\left[\frac{x-\sum_{\ell \in \mathcal{L}_{\text {in }}^{(i)}(n)}\left(\gamma_{\ell}^{(i)}-\lambda_{\ell}^{(i)}\right)}{w_{n}^{(i)}}\right]^{\frac{\beta_{n}^{(i)}}{\beta_{n}^{(i)}-1}}\right) .
\end{aligned}
$$

Let $d_{n}^{(i)}(x)$ denote the summation over $\ell \in \mathcal{L}_{\text {out }}(n)$, and $e_{n}^{(i)}(x)$ the remaining $\log$ term. It can be easily verified that function $h_{n}^{(i)}(x)$ is strictly increasing for $x>\Gamma_{n}^{(i)}$. Therefore, there is a unique $\mu_{n}^{(i) *}$, which can be readily obtained via a root finding method such as the bisection method.

Now, consider the case where $n \notin \mathcal{S}_{i}$. Then, the derivative of the objective in (62) w.r.t $\tilde{r}_{\ell}^{(i)}, \ell \in \mathcal{L}_{\text {in }}^{(i)}(n)$, is given by

$$
\frac{\partial f_{n}^{(i)}\left(\tilde{s}_{n}^{(i)},\left\{\tilde{r}_{\ell}^{(i)}\right\}\right)}{\partial \tilde{r}_{\ell}^{(i)}}=\gamma_{\ell}^{(i)}-\lambda_{\ell}^{(i)}-\frac{\mu_{n}^{(i) *} e^{\tilde{r}_{\ell}^{(i)}}}{\sum_{\ell \in \mathcal{L}_{\mathrm{in}}^{(i)}(n)} e^{\tilde{r}_{\ell}^{(i)}}},
$$

For those $\ell \in \mathcal{L}_{\text {in }}^{(i)}(n)$ for which $\gamma_{\ell}^{(i)}=\lambda_{\ell}^{(i)}$, assume first $\mu_{n}^{(i) *}=0$. Then, any $\tilde{r}_{\ell}^{(i) *}$ that satisfies primal feasibility is acceptable. On the other hand, $\mu_{n}^{(i) *}>0$ implies $\tilde{r}_{\ell}^{(i) *}=-\infty$. For the rest of $\ell \in \mathcal{L}_{\text {in }}^{(i)}(n)$ for which $\gamma_{\ell}^{(i)}>\lambda_{\ell}^{(i)}, \mu_{n}^{(i) *}=0$ implies $\tilde{r}_{\ell}^{(i) *}=\infty$, which violates the primal feasibility. Thus, $\mu_{n}^{(i) *}>0$ holds provided that there exist some $\ell \in \mathcal{L}_{\text {in }}^{(i)}(n)$ such that $\gamma_{\ell}^{(i)}>\lambda_{\ell}^{(i)}$. In the latter case, setting (72) to zero yields

$$
\begin{aligned}
e^{\tilde{r}_{\ell}^{(i) *}}=\left(\frac{\gamma_{\ell}^{(i)}-\lambda_{\ell}^{(i)}}{\mu_{n}^{(i) *}}\right) \sum_{\ell \in \mathcal{L}_{\mathrm{in}}^{(i)}(n)} e^{\tilde{r}_{\ell}^{(i) * *}}, & \gamma_{\ell}^{(i)}>\lambda_{\ell}^{(i)}, \ell \in \mathcal{L}_{\mathrm{in}}^{(i)}(n) .
\end{aligned}
$$

Summing up both sides of (73) w.r.t. $\ell$ yields

$$
\mu_{n}^{(i) *}=\Gamma_{n}^{(i)} .
$$

Plugging (73) and (74) into (64), $\tilde{r}_{\ell}^{(i) *}$ for $\gamma_{\ell}^{(i)}>\lambda_{\ell}^{(i)}, \ell \in$ $\mathcal{L}_{\text {in }}^{(i)}(n)$, are obtained as (46). Problem (P5) may have multiple solutions if $\gamma_{\ell}^{(i)}=\lambda_{\ell}^{(i)}$ for all $\ell \in \mathcal{L}_{\text {in }}^{(i)}(n)$. In this case, one strategy is to choose identical values for all $\tilde{r}_{\ell}^{(i) *}, \ell \in \mathcal{L}_{\text {in }}^{(i)}(n)$, to satisfy $g_{n}^{(i)}\left(\tilde{\mathbf{s}}_{n}^{*}, \tilde{\mathbf{r}}_{n}^{*}, \hat{\mathbf{v}}_{n}^{*}\right)=0$. This leads to (46).

\section{REFERENCES}

[1] M. Avriel and A. C. Williams, "Complementary geometric programming," SIAM J. Appl. Math., vol. 19, no. 1, pp. 125-141, Jul. 1970.

[2] D. O. Awduche, "MPLS and traffic engineering in IP networks," IEEE Commun. Mag., vol. 37, pp. 42-47, Dec. 1999.

[3] D. P. Bertsekas, Nonlinear Programming, 2nd ed. Belmont, MA: Athena Scientific, 1999.

[4] D. P. Bertsekas and R. Gallager, Data Networks. Englewood Cliffs, NJ: Prentice-Hall, 1987.

[5] S. Boyd and L. Vandenberghe, Convex Optimization. Cambridge, U.K.: Cambridge Univ. Press, 2004.

[6] M. Cao, X. Wang, S.-J. Kim, and M. Madihian, "Multi-hop wireless backhaul networks: A cross-layer design paradigm," J. Sel. Areas Commun., vol. 25, no. 4, pp. 738-748, May 2007.

[7] L. Chen, S. H. Low, M. Chiang, and J. C. Doyle, "Cross-layer congestion control, routing and scheduling design in ad hoc wireless networks," in Proc. INFOCOM Conf., Apr. 2006, pp. 1-13.

[8] M. Chiang, S. H. Low, A. R. Calderbank, and J. C. Doyle, "Layering as optimization decomposition: A mathematical theory of network architectures," Proc. IEEE, vol. 95, no. 1, pp. 255-312, Jan. 2007.

[9] M. Chiang, C. W. Tan, D. P. Palomar, D. O'Neill, and D. Julian, "Power control by geometric programming," IEEE Trans. Wireless Commun., vol. 6 , no. 7, pp. 2640-2651, Jul. 2007

[10] B. Fortz, J. Rexford, and M. Thorup, "Traffic engineering with traditional IP routing protocols," IEEE Commun. Mag., vol. 40, no. 10, pp. $118-124$, Oct. 2002

[11] L. Georgiadis, M. J. Neely, and L. Tassiulas, Resource Allocation and Cross-Layer Control in Wireless Networks, ser. Foundations and Trends in Networking. Delft, The Netherlands: Now Publishers, 2006.

[12] S. Ghez, S. Verdú, and S. Schwartz, "Stability properties of slotted ALOHA with multipacket reception capability," IEEE Trans. Autom. Control, vol. 33, no. 7, pp. 640-649, Jul. 1988. 
[13] S.-J. Kim, X. Wang, and M. Madihian, "Distributed joint routing and medium access control for lifetime maximization of wireless sensor networks," IEEE Trans. Wireless Commun., vol. 6, no. 7, pp. 2669-2677, Jul. 2007.

[14] J.-W. Lee, M. Chiang, and A. R. Calderbank, "Jointly optimal congestion and contention control based on network utility maximization," IEEE Commun. Let., vol. 10, no. 3, pp. 216-218, Mar. 2006.

[15] J.-W. Lee, M. Chiang, and A. R. Calderbank, "Utility-optimal random-access control," IEEE Trans. Wireless Commun., vol. 6, no. 7, pp. 2741-2751, Jul. 2007.

[16] J. Liu, A. L. Stolyar, M. Chiang, and H. V. Poor, "Queue back-pressure random access in multi-hop wireless networks: Optimality and stability," IEEE Trans. Inf. Theory, vol. 55, no. 9, pp. 4087-4098, Sep. 2009.

[17] S. H. Low and D. E. Lapsley, "Optimization flow control-I: Basic algorithm and convergence," IEEE/ACM Trans. Netw., vol. 7, no. 6, pp. 861-874, Dec. 1999

[18] R. Madan, S. Cui, S. Lall, and A. Goldsmith, "Cross-layer design for lifetime maximization in interference-limited wireless sensor networks," IEEE Trans. Wireless Commun., vol. 5, no. 11, pp. 3142-3152, Nov. 2006.

[19] M. Mardani, S.-J. Kim, and G. B. Giannakis, "Optimization of wireless multi-hop networks with random access," in Proc. IEEE Workshop on Signal Process. Adv. in Wireless Commun., San Francisco, CA, Jun. 2011.

[20] B. R. Marks and G. P. Wright, "A general inner approximation algorithm for nonconvex mathematical programs," J. Oper. Res. Soc., vol. 26, no. 4, pp. 681-683, Jul. 1978.

[21] J. Mo and J. Walrand, "Fair end-to-end window based congestion control," IEEE/ACM Trans. Netw., vol. 8, no. 5, pp. 556-567, Oct. 2000.

[22] A. H. Mohsenian-Rad, J. Huang, M. Chiang, and V. S. Wong, "Utilityoptimal random access: Reduced complexity, fast convergence, and robust performance," IEEE Trans. Wireless Commun., vol. 8, no. 2, pp. 898-911, Feb. 2009.

[23] T. Nandagopal, T.-E. Kim, X. Gao, and V. Bharghavan, “Achieving MAC layer fairness in wireless packet networks," in Proc. MOBICOM Conf., Boston, MA, Aug. 2000, pp. 87-98.

[24] F. L. Presti, "Joint congestion control, routing and media access control optimization via dual decomposition for ad hoc wireless networks," in Proc. Int. Symp. Model., Anal., Simul. of Wireless Mobile Syst., Montreal, QC, Canada, Oct. 2005, pp. 298-306.

[25] K. Rajawat, N. Gatsis, S.-J. Kim, and G. B. Giannakis, "Cross-layer optimization of coded wireless multi-hop random access networks," IEEE J. Sel. Areas Commun., vol. 29, no. 10, pp. 1970-1980, Dec. 2011.

[26] S. Shakkottai, T. S. Rappaport, and P. C. Karlsson, "Cross-layer design for wireless networks," IEEE Commun. Mag., vol. 41, no. 10, pp. 74-80, Oct. 2003

[27] N. Z. Shor, Minimization Methods for Non-Differentiable Functions. New York: Springer-Verlag, 1985.

[28] S. Supittayapornpong and P. Saengudomlert, "Joint flow control, routing and medium access control in random access multi-hop wireless networks," in Proc. IEEE Int. Conf. Commun. (ICC), Dresden, Germany, Jun. 2009, pp. 1-6.

[29] X. Wang and K. Kar, "Cross-layer rate optimization for proportional fairness in multihop wireless networks with random access," IEEE $J$. Sel. Areas Commun., vol. 24, no. 8, pp. 1548-1559, Aug. 2006.

[30] L. Xiao, M. Johansson, and S. P. Boyd, "Simultaneous routing and resource allocation via dual decomposition," IEEE Trans. Commun., vol. 52, no. 7, pp. 1136-1144, Jul. 2004.

[31] Y. Yu and G. B. Giannakis, "Cross-layer congestion and contention control for wireless ad hoc networks," IEEE Trans. Wireless Commun., vol. 7, no. 1, pp. 37-42, Jan. 2008.

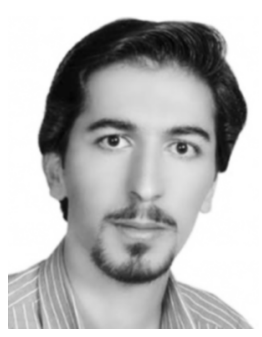

Morteza Mardani (S'06) received the B.Sc. degree in electrical engineering from the Shahid Bahonar University of Kerman, Kerman, Iran, in 2006 and the M.Sc. degree in electrical engineering from the University of Tehran, Tehran, Iran, in 2009.

Since September 2009, he has been working toward the Ph.D. degree with the Department of Electrical and Computer Engineering, University of Minnesota, Minneapolis. His research interests include network inference and optimization, sparse and low rank recovery, and cross-layer design of wireless net-

works.

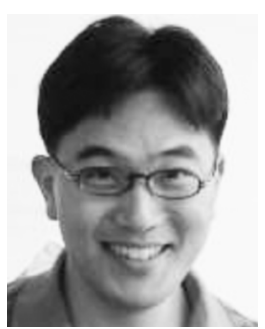

Seung-Jun Kim (M'07) received the B.S. and M.S degrees from Seoul National University, Seoul, Korea, in 1996 and 1998, respectively, and the Ph.D. degree from the University of California at Santa Barbara in 2005, all in electrical engineering.

From 2005 to 2008 , he worked as a research staff member for NEC Laboratories America, Princeton, NJ. Since 2008, he has been with the Department of Electrical and Computer Engineering at the University of Minnesota, where he is currently a Research Assistant Professor. His research interests lie in applying signal processing, optimization, and learning techniques to various domains including wireless communication and networking, and smart power grids.

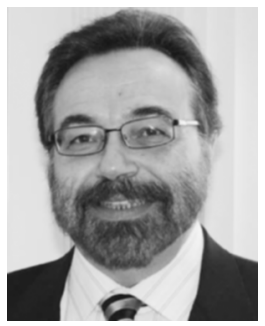

Georgios B. Giannakis (F'97) received the Diploma in electrical engineering from the National Technical University of Athens, Greece, in 1981. From 1982 to 1986, he was with the University of Southern California (USC), where he received the M.Sc. degree in electrical engineering in 1983, the M.Sc. degree in mathematics in 1986, and the Ph.D. degree in electrical engineering in 1986.

Since 1999, he has been a Professor with the University of Minnesota, where he now holds an ADC Chair in Wireless Telecommunications in the Electrical and Computer Engineering Department and serves as Director of the Digital Technology Center. His general interests span the areas of communications, networking and statistical signal processing - subjects on which he has published more than 300 journal papers, 500 conference papers, 20 book chapters, two edited books, and two research monographs. Current research focuses on compressive sensing, cognitive radios, cross-layer designs, wireless sensors, social and power grid networks.

Dri. Giannakis is the (co-) inventor of 21 patents issued, and the (co-) recipient of eight best paper awards from the IEEE Signal Processing (SP) and Communications Societies, including the G. Marconi Prize Paper Award in Wireless Communications. He also received Technical Achievement Awards from the SP Society (2000), from EURASIP (2005), a Young Faculty Teaching Award, and the G. W. Taylor Award for Distinguished Research from the University of Minnesota. He is a Fellow of EURASIP, and has served the IEEE in a number of posts, including that of a Distinguished Lecturer for the IEEE-SP Society. 